\title{
Seychelles: Third Review under the Extended Arrangement, Request for Modification of Performance Criteria, Financing Assurances Review_Staff Report; Staff; Press Release on the Executive Board Discussion.
}

In the context of the third review under the extended arrangement, request for modification of performance criteria, financing assurances review, the following documents have been released and are included in this package:

- $\quad$ The staff report for the Third Review under the Extended Arrangement, Request for Modification of Performance Criteria, Financing Assurances Review, prepared by a staff team of the IMF, following discussions that ended on March 17, 2011, with the officials of Seychelles on economic developments and policies. Based on information available at the time of these discussions, the staff report was completed on May 18, 2011. The views expressed in the staff report are those of the staff team and do not necessarily reflect the views of the Executive Board of the IMF.

- $\quad$ A Press Release summarizing the views of the Executive Board as expressed during its discussion of the staff report that completed the request and/or review.

The policy of publication of staff reports and other documents allows for the deletion of market-sensitive information.

Copies of this report are available to the public from

International Monetary Fund $\bullet$ Publication Services

$70019^{\text {th }}$ Street, N.W. • Washington, D.C. 20431

Telephone: (202) 623-7430 • Telefax: (202) 623-7201

E-mail: publications@imf.org Internet: http://www.imf.org

\section{International Monetary Fund \\ Washington, D.C.}




\title{
INTERNATIONAL MONETARY FUND
}

\section{SEYCHELLES}

\section{Third Review Under the Extended Arrangement, Request for Modification of Performance Criteria, Financing Assurances Review ${ }^{1}$}

\author{
Prepared by the African Department \\ (In consultation with other Departments) \\ Approved by Roger Nord and James Roaf
}

May 18, 2011

\section{Executive Summary}

\begin{abstract}
A three-year arrangement under the Extended Fund Facility (EFF) for 225 percent of quota (SDR 19.8 million) was approved by the Executive Board on December 18, 2009. SDR 9.24 million has been disbursed and SDR 3.52 million will be available upon completion of the third review.

The program is on track. All quantitative performance criteria at end-December 2010 were met. The endDecember 2010 structural benchmark relative to the introduction of a value-added tax was met, but the endApril benchmark on amendments to the Financial Institutions Act is expected to be delayed by two months. Staff recommends completion of the third review and modification of performance criteria.
\end{abstract}

In 2010, aided by sound macroeconomic and structural policies, the Seychelles economy recovered strongly from a debt and balance of payment crisis in 2008, followed by last year's global recession. The high growth was driven by exceptionally high foreign direct investments and a rebound in tourism. Inflation remained near zero, and the country continued to rebuild its international reserves and reduce public debt.

However, the global commodities' price shock and the financial difficulties of Air Seychelles have presented the country with new challenges. Rising food and oil prices will push inflation up and exert pressures on external and fiscal balances, while real GDP growth is expected to slow down. Although the government's support to the national airline is necessary to avoid disruptions in tourist flows, it is causing a significant strain on the budget.

In response to external shocks the policy mix under the program has been adjusted without jeopardizing external stability and the path to debt sustainability.

- The target for accumulation of international reserves has been lowered to absorb part of the external shocks. The flexible exchange regime will continue to provide an additional buffer.

- Monetary policy is being tightened and excess liquidity absorbed to contain second round inflationary effects.

- The 2011 primary surplus target has been slightly lowered and spending cuts have been identified to accommodate one-off expenditures, allowing a smooth pass-through from food and oil prices and the temporary support of the national airline. Air Seychelles's restructuring plan is expected to eliminate losses by end-2011.

Financial sector reforms are gaining momentum. The authorities are implementing a number of legal and supervisory measures to strengthen transparency, remove obstacles to financial intermediation and develop capital markets. In line with their objective of reducing the government's imprint on the economy, they are also adopting strategic plans to restructure and/or privatize state-owned financial institutions.

\footnotetext{
${ }^{1}$ Policy discussions were held in Victoria March 4-17, 2011. Staff met with President James Michel, Vice President and Minister of Finance Danny Faure, Governor Pierre Laporte of the Central Bank of Seychelles, other government officials, representatives of parliament, and the private sector. The mission comprised Jean Le Dem (head), Nikoloz Gigineishvili, Gustavo Ramirez (all AFR), and Nkunde Mwase (SPR).
} 
Contents Page

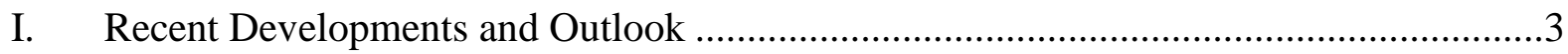

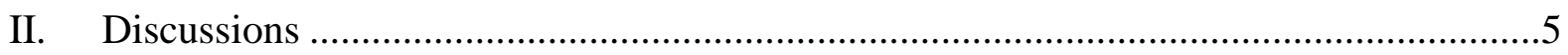

A. Policy Adjustments: Sharing the Burden ...........................................................

B. Structural Reforms .........................................................................................

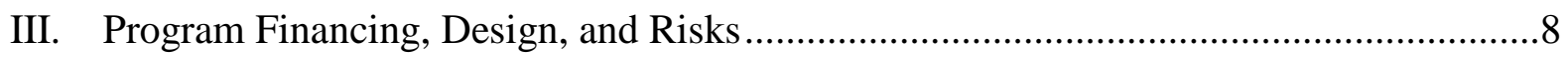

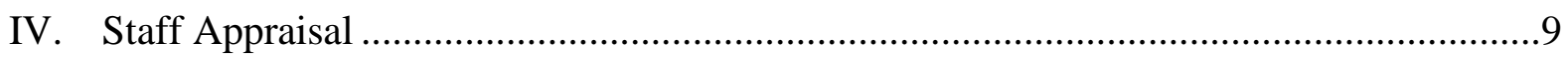

Tables

1. Selected Economic and Financial Indicators, 2007-13 _.............................................13

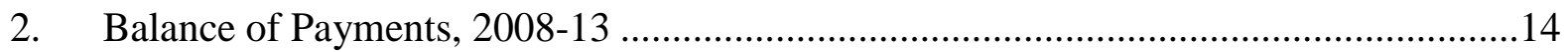

3. Consolidated Government Operations, 2008-13.......................................................15

4. Monetary Survey and Central Bank Accounts, 2008-11 …………………….............19

5. Financial Soundness Indicators for the Banking Sector, 2007-10 .................................18

6. Fund Disbursements and Timing of Reviews Under the Proposed Three-Year EFF, 2009-12 …….......................................................................................19

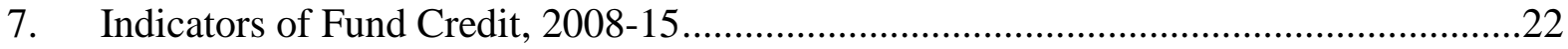

Figures

2. Indicators of Fiscal and Monetary Performance ......................................................12

Annexes

I. Assessing the Adequacy of Seychelles's Gross International ......................................23

II. Transitioning to the GFSM 2001 Fiscal Presentation .................................................25

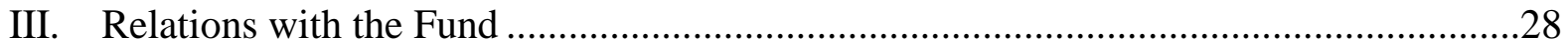

Appendix

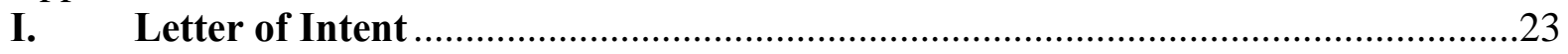

Attachment 1. Supplementary Memorandum of Economic and Financial Policies ....23

Attachment 2. Technical Memorandum of Understanding ..............................................50 


\section{RECENT DEVELOPMENTS AND OUTLOOK}

1. The recovery of the Seychelles economy is rapidly gaining momentum. 2010 marked a reversal from the weak economic performance of the previous two years, when the country suffered from a severe debt crisis in 2008 followed by the global financial crisis in 2009. Real GDP is estimated to have increased by more than 6 percent in 2010, driven by exceptionally high foreign direct investment (FDI) flows and a rebound in tourism. Economic activity has picked up in other sectors as well, such as construction, telecommunications, manufacturing and professional services. Strong domestic demand led to the widening of the current account deficit to about 50 percent of GDP, mainly reflecting high FDI related imports and a terms of trade deterioration due to rising world prices of commodities and aggressive discounting by hotels and tour operators in the aftermath of the global financial crisis. Nevertheless, large capital inflows allowed the central bank to rebuild its reserve buffer to 2.2 months of imports up from 1.6 months a year before.

2. CPI inflation remained low in 2010. With confidence in the national currency continuing to strengthen, the average nominal exchange rate against both the dollar and the euro appreciated helping to keep inflation down. The real effective exchange rate also appreciated by about 8 percent y-o-y in 2010, but still remained about 20 percent below the mid-2008 level.

\section{Consistent with the authorities' debt reduction objective the fiscal stance remained} tight. The primary fiscal surplus reached 9.1 percent of GDP, exceeding the original budget objective by 2.1 percentage points of GDP (Table 3 ). Total revenues (tax and non-tax) outperformed the budget by 4 percent of. There was an acceleration of capital outlays at the end of 2010, but overall, the government saved about half of revenues in excess of the initial budget projections, in line with program understandings. Owing to successful restructuring of external debt coupled with fiscal surpluses total public debt declined from 128.6 percent of GDP in 2009 to 82.3 percent in 2010 .

\section{The benign inflationary environment allowed the central bank to maintain an} accommodative monetary stance. Reserve money grew in line with program projections, driven primarily by the central bank's accumulation of international reserves. Ample liquidity in the banking system pushed interest rates on 90-day government securities further down to below 0.5 percent. Retail lending rates also edged down, and credit to the economy increased by about 14 percent, reaching 27 percent of GDP. ${ }^{2}$

5. The program is on track. All quantitative performance criteria (PCs) at end-December 2010 and indicative targets for end-March were met with margins. The VAT bill was timely submitted to the National Assembly and adopted on December 21, 2010 (end-December 2010

\footnotetext{
${ }^{2}$ The credit growth figure excludes the impact of the reclassification of about SR 200 million in July 2010 from foreign assets to loans.
} 
SB). The end-April 2011 benchmark on amendments to the Financial Institutions Act is being delayed to June to allow more time for the backlogged Attorney General's office to clear the draft bill.

Quantitative Performance Criteria Under the Extended Arrangement, December 2009-March 2011 (Millions of Seychelles rupees; end-of-period)

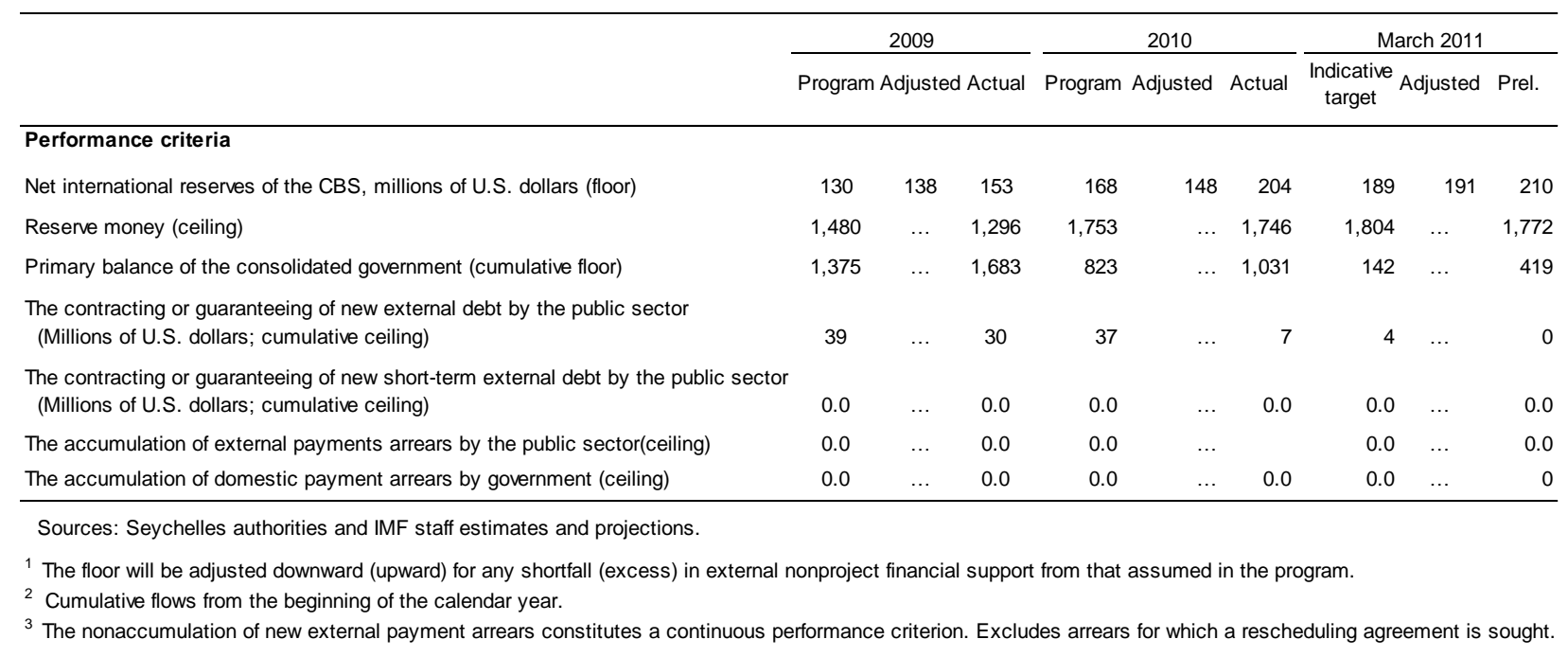

6. Public external debt restructuring has advanced further. In line with the Fund's lending into arrears policy, Seychelles continued good faith negotiations with the remaining creditors. It remains in discussions with two bilateral non-Paris Club creditors (Abu Dhabi and India) on the restructuring of outstanding claims and expects to conclude agreements with the last two remaining commercial creditors shortly_once minor technical issues are settled.

\section{The outlook remains positive despite a worsening external environment. Real GDP} growth is projected to slow down to about 4 percent in 2011, reflecting mainly the projected sharp decline in FDI due to completion of several major residential and hotel construction projects, as well as the impact of higher oil prices. Strong tourism growth, however, should partly offset the impact of rising commodity prices on the external balance. The current account deficit in 2011 is now expected to be five percentage points of GDP wider than previously projected, but will still narrow considerably from 2010 as import demand declines with FDI. Official external reserves are projected to reach 2.4 months of imports at end-2011.

\section{Possible resurgence of inflation came to the forefront of the authorities' policy}

agenda. Year-to-date CPI inflation has picked up modestly in 2011 reaching 0.7 percent in the first quarter. The existing large stocks of petroleum and basic food products purchased earlier at favorable prices by public suppliers have so far mitigated the impact of rising commodities prices domestic prices. However, once these stocks are exhausted and replenished with higher cost imports, domestic prices are expected to increase. With the first round effects of the price pass-through factored in, the end-year CPI is projected to increase by 5.5 percent. The challenge is to contain the second round effects. 
9. The financial situation of Air Seychelles has deteriorated sharply in $\mathbf{2 0 1 0}$ partly due to the increase in fuel costs, but also because of inadequate management. To cover the accumulated losses and pay its suppliers through end-March 2011, the airline borrowed SR 225 million (about 2 percent of GDP) from the state-owned Nouvobanq under a State guarantee. In the absence of measures, losses are projected to amount to 0.2 percent of annual GDP per month.

\section{DISCUSSIONS}

10. Policy discussions for the review focused on: (a) the required monetary and fiscal policy changes to adjust to the commodity price shocks; and (b) key elements of the authorities' administrative and policy reform agenda, in particular financial sector reforms.

\section{A. Policy Adjustments: Sharing the Burden}

11. Discussions focused on how to minimize the cost of adjustment to shocks. The authorities and the mission considered changes in the policy mix that could help the economy absorb the external shocks while preserving hard-won stabilization gains and maintaining the programmed path to debt sustainability. They discussed ways to ensure that the fiscal cushioning of the shocks is rapidly reverted.

\section{Foreign Exchange Policy: Buffering the Shocks}

12. The floating exchange regime will be maintained. The staff concurred with the authorities that a flexible exchange rate has been critical in adjusting to shocks in the past, and if needed, could serve the same purpose in the future. As before, the rupee will float and interventions in the foreign exchange market will be limited only to smoothing out excessive fluctuations.

\section{Part of the required adjustment will come from international reserves. The} authorities maintain the objective of accumulating the equivalent of three months of imports in the medium term, an adequate level for a small island economy with a flexible exchange regime according to a cross-country analysis of reserve adequacy summarized in Annex 1. In view of the worsening external environment, however, it was agreed that NIR overperformance in the 2010 program should not be locked in and that reserves should be allowed to absorb part of the external shocks. Although the path of reserves accumulation has been lowered, expressed in months of prospective imports, an upward trend is maintained.

\section{Monetary Policy: Refocusing on Inflation}

\section{Monetary policy is being tightened to contain inflation expectations and limit the} second round effects of the food and fuel price shocks. The authorities and the mission agreed that the adverse external developments have increased risks to inflation and the exchange rate, and that immediate preemptive measures are needed to prevent disorderly unwinding of the 
liquidity overhang in the banking system. As monthly inflation turned positive in the first few months of 2011, the central bank promptly raised reserve requirements by three percentage points to 13 percent effective April $1 .^{3}$ In addition, the government has started to gradually transfer Social Security Fund deposits with commercial banks to the central bank. These measures are draining excess liquidity (Figure 2) while leaving sufficient precautionary reserves at the banks' disposal. Moreover, should inflationary threats persist, the CBS is prepared to absorb more liquidity by using its market instruments such as repurchase agreements, deposit auctions and foreign exchange swaps. As a result, 3 months Treasury bill rates have already risen to 4.5 percent in early May.

\section{Fiscal Policy: Partially Accommodating One-off Outlays}

\section{The authorities have established a domestic price Stabilization Fund (SF) to} temporarily shield households from a portion of the impact of international price shocks. The SF is designed to provide short-term loans to some parastatals to help smooth price adjustment on select sensitive products and services such as cooking gas, utilities and public transportation. Acknowledging the uncertainty about international oil price movements and the resulting fiscal risks, the authorities have expressed commitment not to convert the operations of the SF into permanent price subsidies. Once the full pass-through is realized, the loans are expected to be repaid.

\section{The authorities agreed to cap disbursements of the SF in 2011 and to closely} monitor its use. Out of the SR 120 million (one percent of GDP) that the authorities had set aside in the SF, only SR 50 million (0.4 percent of GDP) has been budgeted in 2011 and no spending has been effected so far (MEFP I12). The SF support to the Public Utilities Company is expected to decline once the electricity tariff adjustment for fuel price variation is reinstated, by end-September. Staff advised that all prices should be adjusted promptly so as to keep the usage of the SF to a minimum and that concerns about the impact on vulnerable segments of the population could best be addressed by strengthening the targeted social assistance scheme.

17. The authorities believe that Air Seychelles is critical for the tourism sector. Because the national company transports more than half the visitors, they felt that despite the 'open skies' policy, it would be difficult for another carrier to fill the established niche of Air Seychelles in a short period of time. The resulting loss in tourist arrivals would be very costly in terms of economic growth and fiscal revenues. Staff cautioned that time is limited if a sound restructuring plan to restore the finances of Air Seychelles is to be developed by year-end. It was agreed that any residual budget support would be temporary and consistent with the authorities' objectives of fiscal consolidation and debt sustainability.

\footnotetext{
${ }^{3}$ Reserve requirements on domestic currency deposits were increased as of April 1, while those on foreign currency deposits will be gradually raised to the same level by end-June.
} 
18. The ongoing restructuring of Air Seychelles is projected to require transfer of about 0.7 percent of GDP. To stem the further deterioration in the airline's finances, the government and the company's new management agreed on a restructuring plan that includes revising the airfare structure, renegotiating agreements with several partners, eliminating non-profitable routes and downsizing the fleet. If successfully implemented, the plan is projected to reduce losses in the remainder of the year to SR 83 million. The company's financial statements will be monitored on a quarterly basis to ensure that the budget exposure is contained (MEFP III14, 55).

19. A combination of spending cuts and fiscal easing in the second half of 2011 has been agreed upon to partly accommodate the fiscal cost of the SF and the national airline bailout. Additional revenues of 0.2 percent of GDP from delayed dividend payments by the tuna cannery, and a freeze in non-priority domestically financed capital spending of 0.4 percent of GDP will offset the costs of temporary price stabilization. The 2011 primary surplus target has been revised down by 0.5 percent of GDP to 4.5 percent.

\section{B. Structural Reforms}

20. Discussions of the structural reform agenda focused on the strategy to develop and strengthen the financial sector, the ongoing modernization of the tax policy and of the tax and customs administrations, and plans to improve public finance and central bank management.

21. Following the completion of several studies and audit reports, the authorities are adopting a multi-pronged approach to strengthen the financial sector and reduce the role of the state. Based on a study by the CBS, they have identified a number of measures that would help increase transparency and remove obstacles to banking intermediation. These include the introduction of higher disclosure standards, the reduction or elimination of statutory costs related to collateral registration and loan refinancing, and streamlining the litigation and dispute resolution processes. Amendments to the Financial Institutions Act, to be approved by Cabinet by June 2011, will establish the legal basis for new financial products and the creation of credit bureaus. Banking supervision will continue to develop further and move toward a risk-based framework. In addition, the authorities are exploring the development of a stock exchange with a view to developing capital markets and alternative savings and investment opportunities.

\section{The authorities' strategy for restructuring state-owned banks is shaping up}

rapidly. They support IFC's proposed equity participation and its leading role in the privatization of Nouvobanq, as well as its advisory function for restructuring Seychelles Savings Bank (SSB). Meanwhile, they decided to offer 40 percent of SSB shares to bank employees and account holders. Depending on the outcome, more shares of SSB may be sold in the future to the public.

23. The non-bank state financial institutions will also be revamped. Staff expressed concerns about the recent uncontrolled expansion of credit granted by the Development Bank of Seychelles (DBS) at below-market rates and the parallel recourse to government-guaranteed bonds to finance this expansion. In response, the authorities decided to accelerate the 
implementation of the recommendations of a recent technical assistance financed by the FIRST initiative. The mandate of DBS will be redefined more narrowly to cover the financial needs of start-up businesses and small and medium-sized enterprises - a relatively high risk segment that commercial banks are reluctant to enter. The authorities are also studying recommendations of the FIRST initiative experts regarding the Housing Finance Company (HFC), which combines subsidized housing finance, real estate development, and social housing rental functions, and dominates the mortgage market. The action plan for the HFC is being developed in parallel.

24. Tax policy and tax administration. The planned introduction of VAT in mid-2012 is well on track. After passing the VAT legislation in December 2010, the authorities are finalizing the VAT design and have hired a resident TA advisor to assist them in actual implementation over the period of 18 months (MEFP, I15-17). Reforms at the Seychelles Revenue Commission are continuing with Fund technical assistance. Priority is now being given to customs: the new customs management act is expected to be passed in June and to become effective February 2012, once relevant regulations and procedures have been revised (MEFP, I[18-20).

25. Public finance management continues to be strengthened through the implementation of an ambitious action plan. The preparation of a new public finance act, the revision of financial instructions, and the preparation of a new chart of accounts MEFP (II23-24) is under way. Medium-term policy-based budgeting will be introduced in the preparation of the 2012 budget for education and health. To strengthen expenditure control, the Ministry of Finance has started monitoring line ministries' creditor lists on a monthly basis, to continue to ensure the nonaccumulation of domestic payment arrears. The Ministry of Finance and PUC are currently engaged in an extensive exercise to verify the utility consumption of line ministries for 2010 and settle any final bills within 30 days of their submission.

26. Central bank management. The authorities are strengthening the legal framework for central banking, addressing the recommendations of the 2010 updated safeguards assessment (MEFP II38). Internal control and audit mechanisms are also improved (MEFP II39).

\section{Program Financing, Design, And Risks}

27. The program for $\mathbf{2 0 1 1}$ is financed. The residual financing gap for 2011 is expected to be closed by restructuring the rest of public external debt.

28. Quantitative performance criteria and indicative targets under the program for 2011 have been revised in line with updated macroeconomic projections. The proposed new targets for net international reserves, reserve money and fiscal primary surplus reflect the policy adjustments that the authorities intend to adopt in response to the food and fuel price shocks (MEFP table 1).

\section{The attached Technical Memorandum of Understanding has been modified to} clarify the conditionality on domestic arrears and strengthen its monitoring. During the course of 2009-2010, the outstanding stock of government domestic payment arrears at end-2008 (about 3 percentage points of GDP) has been gradually eliminated. However, new minor 
payment delays to the Public Utility Company have emerged late 2010 in one ministry as a result of deficient metering and unbudgeted tariff increases; verifications are under way regarding public utility bills in other ministries. There has not been any net accumulation of domestic arrears so far. Based on an improved definition and a strengthened reporting framework, domestic arrears will be monitored on a monthly basis and their non-accumulation from the beginning of the year will be a quantitative performance criterion.

30. The main risks to the program and the outlook stem from fragility of Air Seychelles and volatility of the external environment. First, should restructuring of the airline take longer than planned and its financial situation deteriorate further, the ensuing quasi-fiscal losses could undermine the authorities' debt reduction objective. Second, the macroeconomic outlook could worsen if international prices for food and fuel continue to increase, leading to additional pressures on external and fiscal accounts. Delayed recovery in Europe - the main source market for tourism - and further depreciation of the euro could also undermine export proceeds and economic activity. And third, the lingering threat of piracy in the Indian Ocean could weaken prospects for tourism and fishing. These risks appear manageable in view of the strong program ownership and track record of policy adjustment to external shocks, broad political support for the reforms, and ongoing international anti-piracy efforts.

\section{Staff Appraisal}

31. The program has made further strides towards its objectives, but the economy faces a new external shock. Growth has recovered from the 2008 debt crisis and the 2009 global recession, international reserves are being steadily rebuilt, and the structural reform agenda is on track. However, a new wave of global commodity price increases is expected to strain the small island economy, stress its external accounts, push up domestic prices from last years' stability, and compound its national airline difficulties. The flow of foreign direct investment that helped the economy rebound is slowing down.

32. The hardly-won stabilization gains put the country in a better position to absorb the shock with minimum adjustment. Some limited fiscal easing is appropriate and the flexible exchange rate regime introduced in 2008 will facilitate the adjustment process. The central bank has adequately reacted to the risks of second-round inflationary pressures by reversing the accommodative monetary policy stance and absorbing banks' excess liquidity.

\section{It will be important to ensure that the authorities' wish to limit the excessive} volatility of some sensitive prices and to support the national airline company do not jeopardize the program's objectives. The government should be commended for the passthrough of international petroleum price increases on car fuels. However, the smoothing of the cooking gas prices and electricity and public bus rates needs to be time-bound to avoid further fiscal drain. The government has mandated the new management of Air Seychelles to restore profitability by year-end. This will require bold measures to limit government cash infusion, 
including downsizing and the elimination of non-profitable routes. The government should also seek private participation to secure the company's future.

34. Staff welcomes the planned additional steps toward the modernization of the tax system and public financial management. The passing of the VAT law and the preparation of its introduction in mid-2012 opens the way for further tax and especially customs administration reforms. Efforts are needed to meet the structural reforms' deadlines for the adoption of the legislative and regulatory framework for budget preparation, execution and reporting.

35. The government should be praised for its decision to speed-up financial sector reforms. Increasing transparency and competition in the banking sector and developing new long-term instruments and a yield curve are welcome steps to increase the role of the private sector in the financing of the economy. The privatization of the two state-owned banks is well underway with the assistance of the IFC and the role of the Development Bank is being rescaled to meet the financing needs of small and medium term enterprises. Further important steps will be to reform housing policy and the social security system.

36. In conclusion, the second phase of the program to set the stage for high sustainable growth is well engaged provided several critical structural reforms are timely

implemented. Staff supports the modification of several quantitative performance criteria for end-June and end-December 2011 and the completion of the third EFF program and financing assurance reviews. 
Figure 1. Seychelles: Indicators of Economic Performance

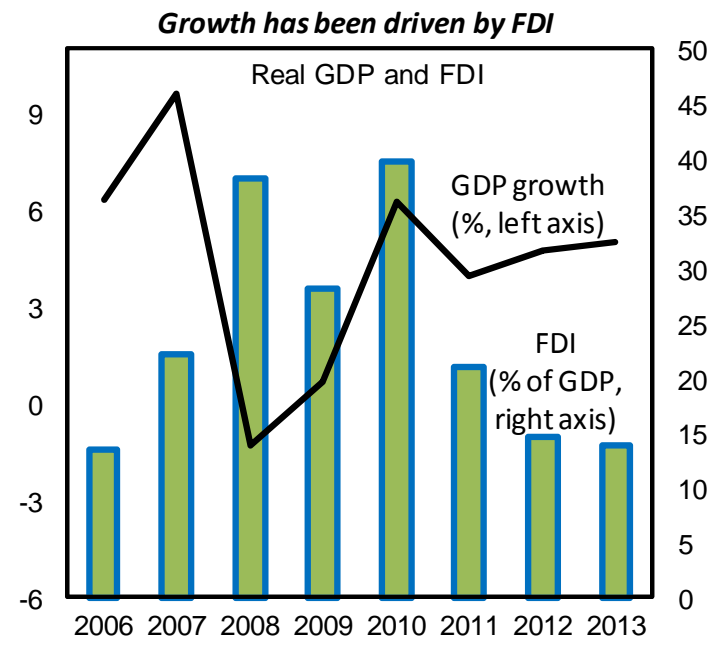

International oil and food prices have not yet passed through to domestic CPI...

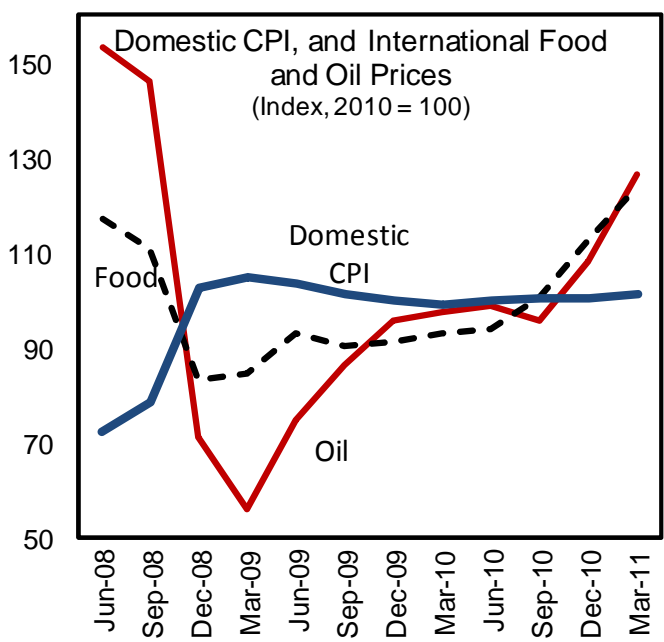

Despite external shocks, the CA will

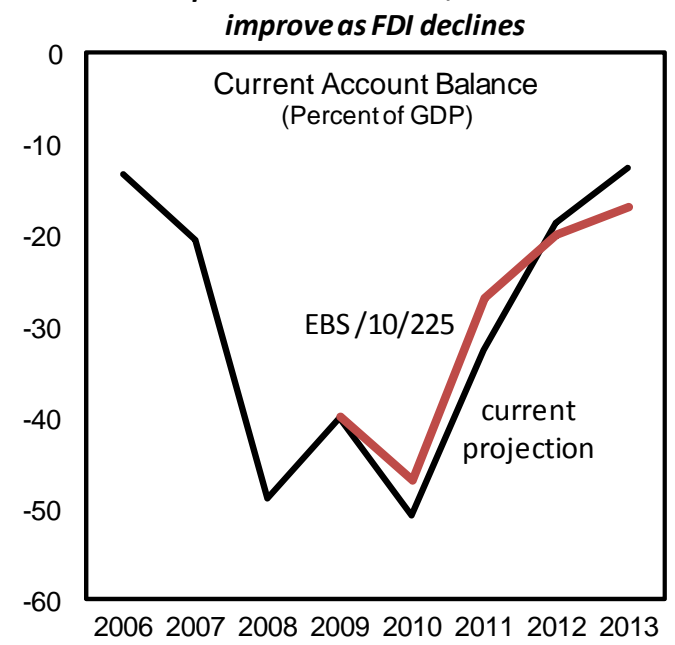

20062007200820092010201120122013

Source: Seychelles authorities, and IMF staff estimates.

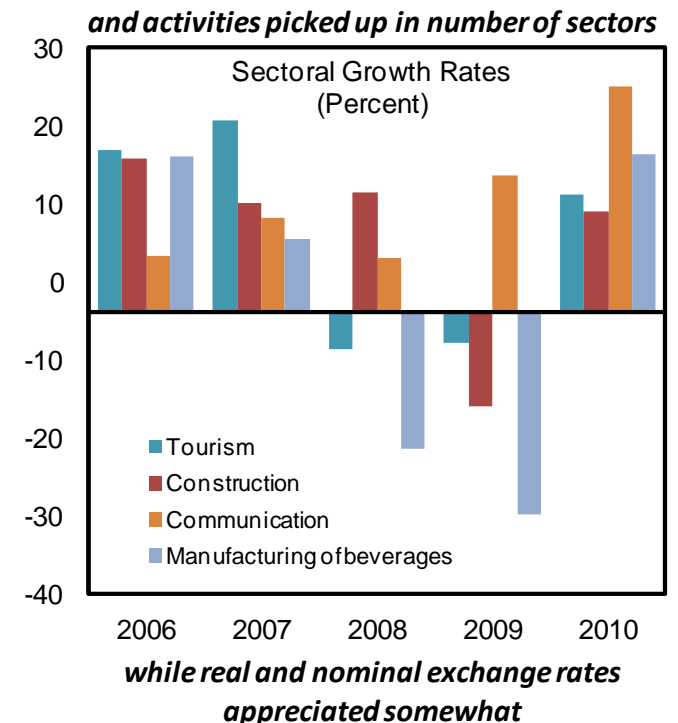
appreciated somewhat

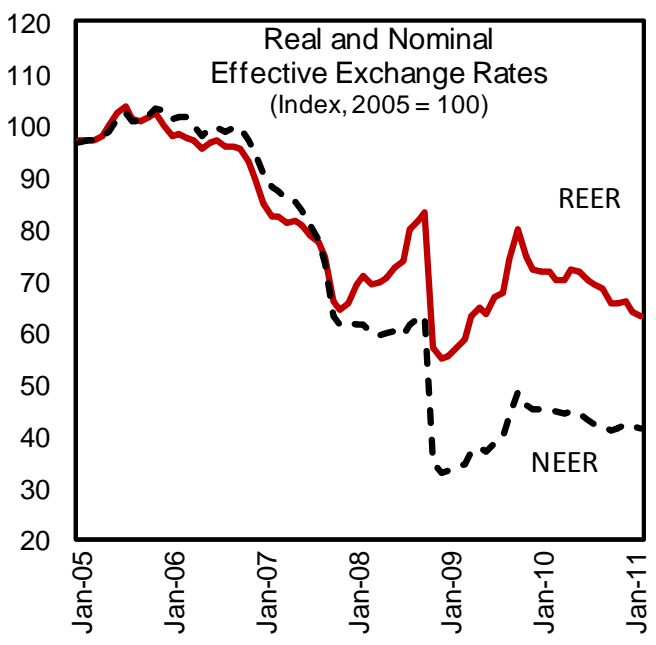

...and international reserves will continue to build-up though at a slower pace

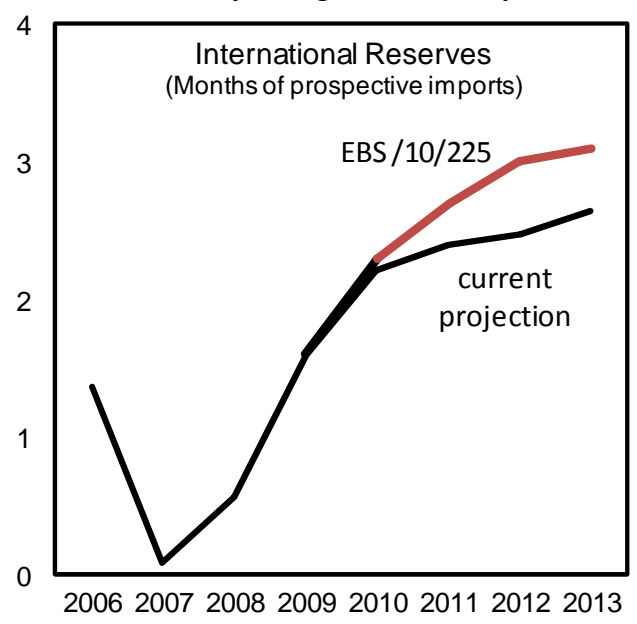


Figure 2. Seychelles: Indicators of Fiscal and Monetary Performance

Prudent fiscal management has led to large surpluses

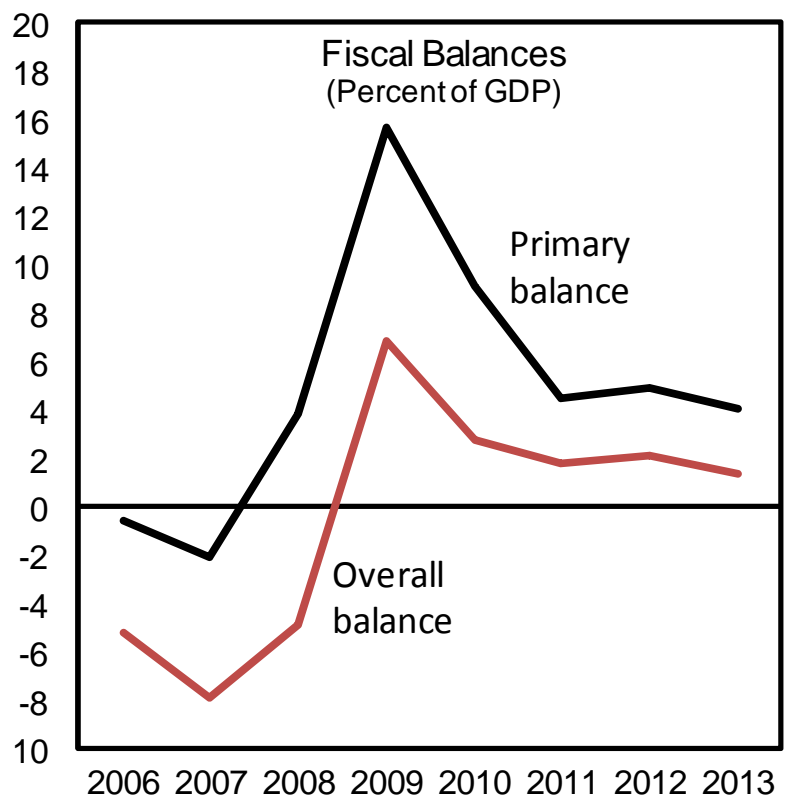

with macroeconomic stabilization interest rates declined.
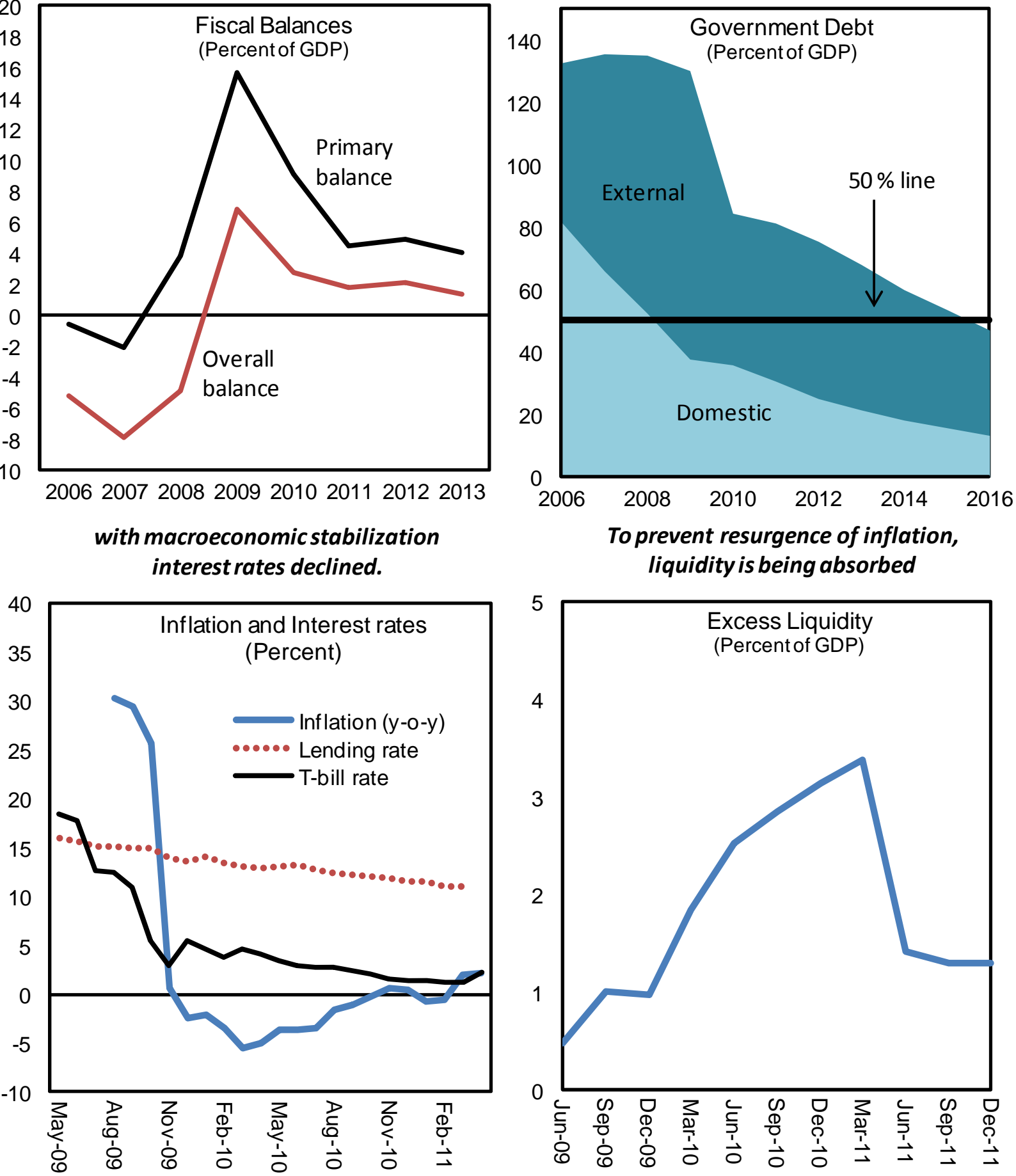

Sources: Seychelles authorities and IMF staff estimates. 
Table 1. Seychelles: Selected Economic and Financial Indicators, 2008-13

\begin{tabular}{|c|c|c|c|c|c|c|c|c|}
\hline & \multirow[t]{2}{*}{2008} & \multirow[t]{2}{*}{2009} & \multicolumn{2}{|c|}{2010} & \multicolumn{2}{|c|}{2011} & \multirow{2}{*}{$\begin{array}{l}2012 \\
\text { Proj. }\end{array}$} & \multirow{2}{*}{$\begin{array}{l}2013 \\
\text { Proj. }\end{array}$} \\
\hline & & & $\begin{array}{c}\text { EBS/10/ } \\
225\end{array}$ & Est. & $\begin{array}{c}\text { EBS/10/ } \\
225\end{array}$ & Proj. & & \\
\hline National income and prices & \multicolumn{8}{|c|}{ (Percentage change, unless otherwise indicated) } \\
\hline Nominal GDP (millions of Seychelles rupees) & 8,709 & 10,725 & 11,303 & 11,294 & 12,000 & 12,016 & 12,953 & 14,064 \\
\hline Real GDP & -1.3 & 0.7 & 6.2 & 6.2 & 4.3 & 4.0 & 4.7 & 5.0 \\
\hline CPI (annual average) & 37.0 & 31.9 & -2.2 & -2.4 & 2.5 & 3.1 & 4.3 & 3.3 \\
\hline CPI (end-of-period) & 63.3 & -2.5 & 1.2 & 0.4 & 3.0 & 5.5 & 3.5 & 2.6 \\
\hline GDP deflator average & 29.3 & 22.3 & -0.8 & -0.9 & 1.8 & 2.3 & 2.9 & 3.4 \\
\hline Money and credit & \multicolumn{8}{|c|}{ (Percentage change, unless otherwise indicated) } \\
\hline Net claims on private sector & 48.6 & -11.4 & 21.5 & 21.4 & 11.3 & 11.9 & $\ldots$ & $\ldots$ \\
\hline Broad money & 29.0 & 7.0 & 12.5 & 13.7 & 11.1 & 5.5 & $\ldots$ & $\ldots$ \\
\hline Reserve money & 0.6 & 15.7 & 35.5 & 34.7 & 5.1 & 1.1 & $\ldots$ & $\ldots$ \\
\hline Velocity (GDP/broad money) & 1.5 & 1.7 & 1.6 & 1.6 & 1.5 & 1.6 & $\ldots$ & $\ldots$ \\
\hline Money multiplier (broad money/reserve money) & 5.3 & 4.9 & 4.1 & 4.2 & 4.3 & 4.4 & $\cdots$ & $\cdots$ \\
\hline Savings-Investment balance & \multicolumn{8}{|c|}{ (In percent of GDP) } \\
\hline External savings & 48.9 & 40.0 & 47.1 & 50.7 & 27.3 & 32.7 & 18.6 & 12.6 \\
\hline Gross national savings & -8.8 & -10.7 & 6.3 & 3.3 & 10.1 & 4.5 & 10.9 & 11.7 \\
\hline Of which: government savings & 7.9 & 8.7 & 7.6 & 8.5 & 13.1 & 12.7 & 10.1 & 9.2 \\
\hline Gross investment & 40.1 & 29.3 & 53.4 & 54.0 & 37.4 & 37.2 & 29.4 & 24.3 \\
\hline Of which: government investment & 2.1 & 5.7 & 8.4 & 9.0 & 10.4 & 10.2 & 7.4 & 7.3 \\
\hline \multicolumn{9}{|l|}{ Government budget } \\
\hline Total revenue, excluding grants & 32.9 & 35.4 & 35.5 & 35.6 & 36.5 & 36.7 & 35.9 & 34.7 \\
\hline Expenditure and net lending & 40.0 & 33.8 & 34.3 & 33.6 & 37.8 & 38.8 & 35.3 & 34.2 \\
\hline Current expenditure & 28.7 & 31.0 & 29.3 & 28.1 & 27.4 & 28.1 & 27.3 & 26.4 \\
\hline Capital expenditure and net lending & 11.2 & 2.7 & 5.0 & 5.5 & 10.5 & 10.8 & 8.0 & 7.8 \\
\hline Overall balance, including grants & -3.4 & 6.0 & 2.7 & 2.8 & 2.7 & 1.8 & 2.1 & 1.4 \\
\hline Primary balance & 3.9 & 15.7 & 9.4 & 9.1 & 5.0 & 4.5 & 5.0 & 4.0 \\
\hline Total public debt ${ }^{1}$ & 135.1 & 128.6 & 76.1 & 82.3 & 71.7 & 76.2 & 69.2 & 61.6 \\
\hline External sector & \multicolumn{8}{|c|}{ (In percent of GDP, unless otherwise indicated) } \\
\hline Current account balance including official transfers & -48.9 & -40.0 & -47.1 & -50.7 & -27.3 & -32.7 & -18.6 & -12.6 \\
\hline Total stock of arrears (millions of U.S. dollars) & 317 & 251 & 33.3 & 30.3 & $\ldots$ & $\ldots$ & $\ldots$ & $\ldots$ \\
\hline Total public external debt outstanding (millions of U.S. dollars) ${ }^{1}$ & 759 & 732 & 425 & 456 & 448 & 489 & 509 & 501 \\
\hline (in percent of GDP) & 82.8 & 92.6 & 45.3 & 48.7 & 46.2 & 50.7 & 50.4 & 46.7 \\
\hline Terms of trade (= - deterioration) & 2.0 & 14.5 & $\cdots$ & -5.6 & $\ldots$ & -9.3 & $\ldots$ & $\ldots$ \\
\hline Real effective exchange rate (average, percent change) & -7.1 & -6.6 & $\cdots$ & 5.4 & $\cdots$ & $\cdots$ & $\ldots$ & $\cdots$ \\
\hline Gross official reserves (end of year, millions of U.S. dollars) & 51 & 169 & 218 & 238 & 253 & 240 & 250 & 272 \\
\hline In months of imports, c.i.f. & 0.6 & 1.6 & 2.3 & 2.2 & 2.7 & 2.4 & 2.5 & 2.6 \\
\hline \multicolumn{9}{|l|}{ Exchange rate } \\
\hline Seychelles rupees per US\$1 (end of period) & 16.6 & 11.2 & $\ldots$ & 12.2 & $\ldots$ & 12.7 & $\ldots$ & $\ldots$ \\
\hline Seychelles rupees per US $\$ 1$ (period average) & 9.5 & 13.6 & $\ldots$ & 12.1 & $\ldots$ & 12.5 & $\ldots$ & $\ldots$ \\
\hline
\end{tabular}

Sources: Central Bank of Seychelles; Ministry of Finance; and IMF staff estimates and projections.

${ }^{1}$ The 2010 number exceeds the previous staff report figure by about 6 percentage points of GDP, reflecting in part the slower-thanprojected progress on the restructuring of the remaining external debt, and some foreign currency debt held by commercial banks, which had been converted to domestic currency debt as part of debt restructuring and had not been recorded. 
Table 2. Seychelles: Balance of Payments, 2008-13

(Millions of U.S. dollars)

\begin{tabular}{|c|c|c|c|c|c|c|c|c|}
\hline & \multirow[t]{2}{*}{2008} & \multirow[t]{2}{*}{2009} & \multicolumn{2}{|c|}{2010} & \multicolumn{2}{|c|}{2011} & 2012 & 2013 \\
\hline & & & $\begin{array}{c}\text { EBS/10/ } \\
225 \\
\end{array}$ & Proj. & $\begin{array}{c}\text { EBS/10/ } \\
225 \\
\end{array}$ & Proj. & Proj. & Proj \\
\hline Current account & -448 & -316 & -441 & -474 & -265 & -316 & -187 & -136 \\
\hline (Percent of GDP) & -48.9 & -40.0 & -47.1 & -50.7 & -27.3 & -32.7 & -18.6 & -12.6 \\
\hline Trade balance & -412 & -253 & -370 & -412 & -222 & -288 & -164 & -113 \\
\hline Exports of goods & 502 & 427 & 412 & 427 & 488 & 531 & 540 & 563 \\
\hline Of which: oil re-exports & 200 & 184 & 141 & 158 & 146 & 215 & 216 & 220 \\
\hline Of which: tuna exports & 216 & 165 & 213 & 210 & 252 & 222 & 224 & 238 \\
\hline Imports of goods & $-1,009$ & -780 & -848 & -895 & -812 & -914 & -844 & -844 \\
\hline Of which: Oil imports & -278 & -209 & -204 & -217 & -204 & -298 & -305 & -312 \\
\hline FDI-related imports & -288 & -220 & -263 & -252 & -165 & -145 & -108 & -109 \\
\hline Other & -390 & -335 & -380 & -426 & -442 & -471 & -407 & -414 \\
\hline Exports of services & 486 & 404 & 413 & 434 & 437 & 467 & 502 & 534 \\
\hline Of which: Tourism earnings & 270 & 208 & 219 & 231 & 231 & 257 & 278 & 298 \\
\hline Imports of services & -391 & -305 & -348 & -378 & -336 & -372 & -362 & -366 \\
\hline Income, net & -94 & -105 & -81 & -83 & -43 & -31 & -31 & -31 \\
\hline Of which: transfers of profits and dividends & -30 & -58 & -29 & -34 & -17 & -14 & -10 & -10 \\
\hline Of which: interest payments due ${ }^{1}$ & -70 & -48 & -50 & -43 & -26 & -17 & -22 & -23 \\
\hline Current transfers, net & 57 & 42 & 10 & 21 & 0 & 4 & 8 & $\varepsilon$ \\
\hline Of which: General government, net & 32 & 40 & 11 & 21 & 12 & 5 & 9 & s \\
\hline Capital and financial account & 119 & 39 & 369 & 394 & 286 & 297 & 189 & 162 \\
\hline Capital account & 5 & 52 & 289 & 275 & 34 & 68 & 10 & 0 \\
\hline Of which: Debt forgiveness & $\ldots$ & -41 & -270 & -267 & 0 & 0 & 0 & $c$ \\
\hline Financial account & 114 & -12 & 80 & 119 & 252 & 228 & 179 & 162 \\
\hline Direct investment, net & 166 & 113 & 284 & 313 & 117 & 143 & 103 & 103 \\
\hline Portfolio investment, net ${ }^{1}$ & -122 & 1 & 1 & 1 & 1 & 1 & 1 & 1 \\
\hline Other investment, net & 70 & -126 & -206 & -195 & 135 & 85 & 76 & 59 \\
\hline Government and government-guaranteed & 19 & -105 & -275 & -273 & 12 & 20 & 17 & 6 \\
\hline Disbursements & 84 & 12 & 41 & 38 & 24 & 36 & 28 & 18 \\
\hline Project loans & 84 & 1 & 13 & 10 & 24 & 36 & 28 & 18 \\
\hline Program loans & 0 & 11 & 27 & 28 & 0 & 0 & 0 & 0 \\
\hline Amortization & -65 & -79 & -46 & -46 & -11 & -14 & -13 & -18 \\
\hline Private sector ${ }^{2}$ & 54 & 7 & 53 & 42 & 112 & 55 & 49 & 43 \\
\hline Of which: Foreign loans for investment & 185 & 110 & 126 & 59 & 113 & 59 & 46 & 46 \\
\hline Net errors and omissions & 115 & 133 & 88 & 26 & 0 & 0 & 0 & 0 \\
\hline Overall balance & -212 & -24 & 15 & -54 & 21 & -19 & 2 & 27 \\
\hline Financing & 212 & 24 & 66 & 54 & -27 & 8 & -9 & -33 \\
\hline Change in net international reserves (increase: - ) & 38 & -43 & -36 & -52 & -27 & 8 & -9 & -33 \\
\hline Change in gross official reserves (increase: -) & 28 & -50 & -49 & -68 & -35 & -3 & -10 & -22 \\
\hline Liabilities to IMF, net & 10 & 8 & 13 & 16 & 8 & 10 & 0 & -11 \\
\hline Other Net Foreign Assets (increase: -) & 0 & -13 & 10 & 10 & 0 & 0 & 0 & 0 \\
\hline Exceptional financing & 174 & 79 & 92 & 97 & 0 & 0 & 0 & 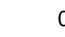 \\
\hline Change in arrears (increase: + ) & 174 & -74 & -162 & -160 & 0 & 0 & 0 & 0 \\
\hline Clearance of arrears & 0 & 153 & 254 & 257 & 0 & 0 & 0 & 0 \\
\hline Financing gap & 0 & 0 & 4 & 0 & 6 & 11 & 7 & 6 \\
\hline \multicolumn{9}{|l|}{ Memorandum items: } \\
\hline Exports G\&S growth, percent & 13.2 & -15.9 & 0.0 & 3.5 & 12.2 & 16.0 & 4.4 & 5.3 \\
\hline Tourism growth, percent & -4.8 & -23.3 & 6.8 & 11.1 & 5.9 & 11.4 & 8.2 & 7.0 \\
\hline Imports G\&S growth, percent & 33.7 & -22.5 & 13.6 & 17.3 & -4.0 & 1.1 & -6.3 & 0.3 \\
\hline Of which: Non-FDI and domestic-oil imports & 6.2 & -16.8 & 4.2 & 29.2 & 5.3 & 5.3 & -2.8 & 1.8 \\
\hline Exports G\&S, percent of GDP & 107.8 & 105.2 & 88.1 & 91.9 & 95.3 & 103.5 & 103.3 & 102.1 \\
\hline Imports G\&S, percent of GDP & 152.8 & 137.2 & 127.6 & 136.0 & 118.2 & 133.4 & 119.6 & 112.6 \\
\hline FDI (incl. loans), USD million & 350 & 223 & 411 & 372 & 230 & 202 & 148 & 148 \\
\hline FDI (incl. loans), percent of GDP & 38.2 & 28.2 & 43.8 & 39.8 & 23.7 & 20.9 & 14.7 & 13.8 \\
\hline Gross international reserves (stock, e.o.p.) & 51 & 169 & 218 & 238 & 253 & 240 & 250 & 272 \\
\hline Months of prospective imports of goods and services & 0.6 & 1.6 & 2.3 & 2.2 & 2.7 & 2.4 & 2.5 & 2.6 \\
\hline Months of non-FDI imports of G\&S excl. oil re-exports & 0.9 & 2.4 & 3.1 & 3.1 & 3.7 & 3.3 & 3.4 & 3.6 \\
\hline Of which: Valuation change (+gain) & & 68 & & $\ldots$ & $\ldots$ & $\ldots$ & $\ldots$ & .. \\
\hline Scheduled public external debt service & 221 & 114 & 98 & 85 & 40 & 27 & 30 & 37 \\
\hline (Percent of exports of goods and services) & 22.4 & 13.7 & 11.9 & 9.9 & 4.3 & 2.7 & 2.9 & 3.4 \\
\hline Public and publicly guaranteed external debt ${ }^{3}$ & 759 & 732 & 425 & 456 & 448 & 489 & 509 & 501 \\
\hline (Percent of GDP) & 82.8 & 92.6 & 45.3 & 48.7 & 46.2 & 50.7 & 50.4 & 46.7 \\
\hline o.w. Publicly guaranteed external debt (percent of GDP) & $\ldots$ & $\ldots$ & $\ldots$ & $\ldots$ & $\ldots$ & 1.9 & 3.8 & 4.6 \\
\hline GDP & 916 & 790 & 937 & 936 & 971 & 965 & 1,008 & 1,074 \\
\hline
\end{tabular}


Table 3. Seychelles: Consolidated Government Operations, 2008-13 ${ }^{1}$

(Millions of Seychelles rupees; cumulative from the start of the year)

\begin{tabular}{|c|c|c|c|c|c|c|c|c|c|c|c|c|c|c|c|}
\hline & \multirow[t]{3}{*}{2008} & \multirow[t]{3}{*}{2009} & \multicolumn{3}{|c|}{2010} & \multicolumn{8}{|c|}{2011} & \multirow{3}{*}{$\begin{array}{l}2012 \\
\text { Proj. }\end{array}$} & \multirow{3}{*}{$\begin{array}{r}2013 \\
\text { Proj }\end{array}$} \\
\hline & & & \multirow{2}{*}{\multicolumn{2}{|c|}{$\begin{array}{c}\text { Budget EBS/10/ } \\
225\end{array}$}} & \multirow[b]{2}{*}{ Prel. } & \multicolumn{2}{|c|}{ Q1 } & \multicolumn{2}{|c|}{ Q2 } & \multicolumn{2}{|c|}{ Q3 } & Q4 & & & \\
\hline & & & & & & Prog & Prel. & Prog & Proj. & Prog & Proj. & Prog & Proj. & & \\
\hline $\begin{array}{l}\text { Total revenue and grants } \\
\text { Total revenue }\end{array}$ & $\begin{array}{l}3,190 \\
2,868\end{array}$ & $\begin{array}{l}\mathbf{4 , 2 6 2} \\
3,793\end{array}$ & $\begin{array}{l}3,866 \\
3,514\end{array}$ & $\begin{array}{l}4,178 \\
4,008\end{array}$ & $\begin{array}{l}4,119 \\
4,018\end{array}$ & $\begin{array}{r}1,091 \\
990\end{array}$ & $\begin{array}{l}\mathbf{1}, \mathbf{1 3 4} \\
1,130\end{array}$ & $\begin{array}{l}2,219 \\
1,981\end{array}$ & $\begin{array}{l}2,339 \\
2,076\end{array}$ & $\begin{array}{l}3,560 \\
3,189\end{array}$ & $\begin{array}{l}3,636 \\
3,251\end{array}$ & $\begin{array}{l}4,856 \\
4,381\end{array}$ & $\begin{array}{l}4,881 \\
4,405\end{array}$ & $\begin{array}{l}4,847 \\
4,644\end{array}$ & $\begin{array}{l}5,004 \\
4,879\end{array}$ \\
\hline $\begin{array}{l}\text { Tax } \\
\quad \text { Personal income tax }\end{array}$ & 2,456 & 3,318 & $\begin{array}{r}3,090 \\
190\end{array}$ & $\begin{array}{r}3,527 \\
181\end{array}$ & $\begin{array}{r}3,544 \\
195\end{array}$ & $\begin{array}{l}918 \\
140\end{array}$ & $\begin{array}{l}990 \\
136\end{array}$ & $\begin{array}{r}1,786 \\
290\end{array}$ & $\begin{array}{r}1,856 \\
290\end{array}$ & $\begin{array}{r}2,815 \\
430\end{array}$ & $\begin{array}{r}2,853 \\
430\end{array}$ & $\begin{array}{r}3,905 \\
573\end{array}$ & $\begin{array}{r}3,905 \\
573\end{array}$ & $\begin{array}{r}4,151 \\
602\end{array}$ & $\begin{array}{r}4,344 \\
653\end{array}$ \\
\hline Social security tax & 295 & 384 & 233 & 220 & 228 & $\ldots$ & $\ldots$ & $\ldots$ & $\ldots$ & $\ldots$ & $\ldots$ & $\ldots$ & $\ldots$ & $\ldots$ & \\
\hline Trade tax & 410 & 437 & $\ldots$ & 369 & 355 & 87 & 95 & 171 & 171 & 264 & 264 & 377 & 377 & 366 & 366 \\
\hline Excise tax & $\ldots$ & $\ldots$ & $\ldots$ & 559 & 580 & 125 & 153 & 251 & 251 & 399 & 399 & 564 & 564 & 606 & 636 \\
\hline Goods and services tax (GST) & 951 & 1,348 & $\ldots$ & 1,078 & 1,050 & 271 & 266 & 567 & 567 & 851 & 851 & 1,216 & 1,216 & 1,277 & 1,365 \\
\hline Business tax & 524 & 802 & 602 & 796 & 790 & 134 & 268 & 271 & 349 & 539 & 579 & 772 & 772 & 832 & 904 \\
\hline Other & 277 & 346 & 286 & 323 & 346 & 148 & 55 & 209 & 202 & 292 & 290 & 350 & 350 & 390 & 336 \\
\hline Pension Fund contribution & $\ldots$ & $\ldots$ & $\ldots$ & $\ldots$ & $\ldots$ & 13 & 18 & 27 & 27 & 40 & 40 & 54 & 54 & 78 & 85 \\
\hline Nontax & 412 & 474 & 424 & 482 & 474 & 72 & 140 & 195 & 219 & 374 & 398 & 477 & 501 & 493 & 535 \\
\hline Fees and charges & 145 & 182 & 195 & 181 & 191 & 52 & 43 & 99 & 99 & 150 & 150 & 199 & 199 & 214 & 232 \\
\hline Dividends from parastatals & 64 & 148 & 129 & 229 & 206 & 9 & 76 & 53 & 81 & 165 & 193 & 207 & 235 & 205 & 223 \\
\hline Other & 203 & 143 & 101 & 72 & 77 & 11 & 20 & 43 & 39 & 59 & 55 & 70 & 66 & 74 & 80 \\
\hline External grants & 321 & 470 & 352 & 170 & 101 & 101 & 4 & 238 & 264 & 371 & 386 & 475 & 476 & 203 & 125 \\
\hline $\begin{array}{l}\text { Expenditure and net lending } \\
\text { Current expenditure }\end{array}$ & $\begin{array}{l}3,483 \\
2,503\end{array}$ & $\begin{array}{l}3,623 \\
3,327\end{array}$ & $\begin{array}{l}3,788 \\
3,244\end{array}$ & $\begin{array}{l}3,878 \\
3,315\end{array}$ & $\begin{array}{l}3,800 \\
3,177\end{array}$ & $\begin{array}{r}1,050 \\
742\end{array}$ & $\begin{array}{l}816 \\
719\end{array}$ & $\begin{array}{l}\mathbf{2 , 1 3 5} \\
1,530\end{array}$ & $\begin{array}{l}2,244 \\
1,590\end{array}$ & $\begin{array}{l}3,273 \\
2,344\end{array}$ & $\begin{array}{l}3,432 \\
2,450\end{array}$ & $\begin{array}{l}4,538 \\
3,283\end{array}$ & $\begin{array}{l}4,667 \\
3,372\end{array}$ & $\begin{array}{l}4,569 \\
3,531\end{array}$ & $\begin{array}{l}4,806 \\
3,710\end{array}$ \\
\hline Primary current expenditure & 1,875 & 2,283 & 2,489 & 2,558 & 2,465 & 641 & 617 & 1,374 & 1,425 & 2,116 & 2,192 & 3,000 & 3,050 & 3,167 & 3,346 \\
\hline Wages and salaries & 688 & 766 & 735 & 694 & 693 & 224 & 208 & 453 & 453 & 679 & 679 & 906 & 906 & 970 & 1,053 \\
\hline Goods and services & 489 & 715 & 777 & 925 & 843 & 169 & 166 & 393 & 393 & 622 & 622 & 973 & 973 & 983 & 1,035 \\
\hline Transfers & 692 & 783 & 966 & 927 & 918 & 246 & 243 & 522 & 572 & 807 & 882 & 1,108 & 1,158 & 1,203 & 1,246 \\
\hline Social program of central government & 192 & 290 & 341 & 224 & 233 & 53 & 46 & 120 & 120 & 180 & 180 & 271 & 271 & 259 & 259 \\
\hline Transfers to public sector from central governmen & 220 & 199 & 273 & 351 & 332 & 90 & 88 & 196 & 246 & 319 & 394 & 426 & 476 & 454 & 473 \\
\hline Benefits and programs of Social Security Fund & 279 & 295 & 352 & 352 & 353 & 84 & 92 & 168 & 168 & 252 & 252 & 337 & 337 & 412 & 432 \\
\hline Benefits Payment of Seychelles Pension Fund & $\ldots$ & $\ldots$ & & & $\cdots$ & 19 & 17 & 37 & 37 & 56 & 56 & 75 & 75 & 78 & 82 \\
\hline Other & 6 & 19 & 12 & 12 & 12 & 2 & 1 & 6 & 7 & 8 & 9 & 12 & 13 & 12 & 12 \\
\hline Interest payments due & 629 & 1,044 & 755 & 759 & 712 & 101 & 102 & 156 & 165 & 228 & 258 & 284 & 322 & 364 & 365 \\
\hline Foreign interest & 344 & 428 & 316 & 411 & 402 & 72 & 56 & 97 & 76 & 140 & 123 & 169 & 145 & 199 & 219 \\
\hline Domestic interest & 285 & 616 & 439 & 348 & 310 & 30 & 46 & 59 & 89 & 88 & 135 & 115 & 177 & 165 & 146 \\
\hline Capital expenditure ${ }^{2}$ & 183 & 608 & 833 & 948 & 1,011 & 307 & 90 & 604 & 625 & 903 & 926 & 1,253 & 1,211 & 965 & 1,027 \\
\hline Net lending & 796 & -315 & -349 & -385 & -389 & 0 & 7 & 1 & 29 & 1 & 57 & 2 & 85 & 73 & 69 \\
\hline Primary balance & 336 & 1,683 & 833 & 1,059 & 1,031 & 142 & 419 & 240 & 260 & 515 & 462 & 602 & 536 & 642 & 562 \\
\hline Overall balance, commitment basis & -293 & 639 & 78 & 301 & 319 & 41 & 318 & 85 & 95 & 287 & 204 & 318 & 214 & 278 & 198 \\
\hline Change in arrears & 31 & 96 & -100 & -41 & -3 & 0 & -8 & 0 & -8 & 0 & -8 & 0 & -8 & 0 & 0 \\
\hline External interest & 167 & 321 & 0 & 59 & 90 & 0 & 0 & 0 & 0 & 0 & 0 & 0 & 0 & 0 & 0 \\
\hline Budget & -136 & -224 & -100 & -100 & -92 & 0 & -8 & 0 & -8 & 0 & -8 & 0 & -8 & 0 & 0 \\
\hline Overall balance, cash basis (after grants) & -262 & 735 & -22 & 259 & 316 & 41 & 310 & 85 & 88 & 287 & 197 & 318 & 206 & 278 & 198 \\
\hline Financing & 262 & -735 & 22 & -259 & -316 & -41 & -310 & -85 & -88 & -287 & -197 & -318 & -206 & -278 & -198 \\
\hline Foreign financing & 233 & -174 & 42 & 70 & 236 & 16 & -12 & 64 & 22 & 93 & 37 & 127 & 56 & -57 & -143 \\
\hline Disbursements & 672 & 137 & 442 & 489 & 584 & 73 & 1 & 145 & 72 & 219 & 145 & 292 & 217 & 109 & 86 \\
\hline Project loans & 672 & 137 & 118 & 162 & 247 & 73 & 1 & 145 & 72 & 219 & 145 & 292 & 217 & 109 & 86 \\
\hline Program/budget support & 0 & 120 & 324 & 328 & 337 & 0 & 0 & 0 & 0 & 0 & 0 & 0 & 0 & 0 & 0 \\
\hline Scheduled amortization & $-1,630$ & $-1,090$ & -413 & -554 & -554 & -56 & -32 & -81 & -69 & -126 & -126 & -166 & -179 & -166 & -229 \\
\hline Change in amortization arrears & 1,191 & $-1,213$ & $-1,945$ & $-2,845$ & $-2,811$ & 0 & 18 & 0 & 18 & 0 & 18 & 0 & 18 & 0 & 0 \\
\hline Clearance of arrears & 0 & 1,938 & 1,945 & 2,980 & 3,017 & 0 & 0 & 0 & 0 & 0 & 0 & 0 & 0 & 0 & 0 \\
\hline Debt service relief & 0 & 56 & 12 & 0 & 0 & 0 & 0 & 0 & 0 & 0 & 0 & 0 & 0 & 0 & 0 \\
\hline Domestic financing, net & -70 & -710 & -733 & -530 & -665 & -132 & -241 & -260 & -130 & -565 & -344 & -666 & -458 & -379 & -173 \\
\hline Bank financing & -100 & -827 & -494 & -282 & -235 & -119 & -196 & -234 & -96 & -508 & -288 & -600 & -391 & -341 & -156 \\
\hline CBS & -3 & -376 & -177 & -151 & -131 & -100 & -18 & -200 & -68 & -250 & -68 & -250 & -68 & 0 & 0 \\
\hline CBS recapitalization & $\ldots$ & $\ldots$ & 188 & 188 & 188 & $\ldots$ & $\ldots$ & $\ldots$ & $\ldots$ & $\ldots$ & $\ldots$ & $\ldots$ & $\ldots$ & $\ldots$ & $\cdots$ \\
\hline Commercial banks & -96 & -451 & -505 & -320 & -291 & -19 & -178 & -34 & -28 & -258 & -220 & -350 & -323 & -341 & -156 \\
\hline Nonbank financing & 30 & 117 & -51 & -248 & -430 & -13 & -45 & -26 & -34 & -56 & -55 & -67 & -67 & -38 & -17 \\
\hline Privatization and long-term lease of fixed assets & 219 & 222 & 152 & 153 & 156 & 37 & 28 & 37 & 65 & 37 & 101 & 146 & 146 & 157 & 118 \\
\hline Statistical discrepancy & -120 & 22 & 52 & 4 & -44 & 0 & -85 & 0 & -85 & 0 & -85 & 0 & -85 & 0 & 0 \\
\hline Fiscal financing gap & 0 & 0 & 510 & 44 & 0 & 38 & 0 & 38 & 41 & 75 & 94 & 75 & 134 & 0 & 0 \\
\hline Memorandum item: & & & & & & & & & & & & & & & \\
\hline External debt service due & 1,974 & 1,519 & 729 & 965 & 957 & 128 & 87 & 178 & 145 & 265 & 249 & 334 & 324 & 365 & 448 \\
\hline Sources: Seychelles auth & & & & & & & & & & & & & & & \\
\hline${ }^{2}$ Includes contingency spendi & & & & & & & & & & & & & & & ed) \\
\hline
\end{tabular}


Table 3. Seychelles: Consolidated Government Operations, $2008-13^{1}$ (continued)

(Percent of GDP; cumulative from the start of the year)

\begin{tabular}{|c|c|c|c|c|c|c|c|c|c|c|c|c|c|c|c|}
\hline & \multirow[t]{3}{*}{2008} & \multirow[t]{3}{*}{2009} & \multirow{2}{*}{\multicolumn{3}{|c|}{2010}} & \multicolumn{8}{|c|}{2011} & \multirow{3}{*}{$\begin{array}{l}2012 \\
\text { Proj. }\end{array}$} & \multirow{3}{*}{$\begin{array}{r}2013 \\
\text { Proj }\end{array}$} \\
\hline & & & & & & \multicolumn{2}{|c|}{ Q1 } & \multicolumn{2}{|c|}{ Q2 } & \multicolumn{2}{|c|}{ Q3 } & \multicolumn{2}{|c|}{ Q4 } & & \\
\hline & & & \multicolumn{2}{|c|}{$\begin{array}{l}\text { Budget EBS/10/ } \\
225\end{array}$} & Prel. & Prog & Prel. & Prog & Proj. & Prog & Proj. & Prog & Proj. & & \\
\hline Total revenue and grants & 36.6 & 39.7 & 34.5 & 37.0 & 36.5 & 9.1 & 9.4 & 18.5 & 19.5 & 29.7 & 30.3 & 40.5 & 40.6 & 37.4 & 35.6 \\
\hline Total revenue & 32.9 & 35.4 & 31.4 & 35.5 & 35.6 & 8.3 & 9.4 & 16.5 & 17.3 & 26.6 & 27.1 & 36.5 & 36.7 & 35.9 & 34.7 \\
\hline Tax & 28.2 & 30.9 & 27.6 & 31.2 & 31.4 & 7.7 & 8.2 & 14.9 & 15.4 & 23.5 & 23.7 & 32.5 & 32.5 & 32.0 & 30.9 \\
\hline Personal income tax & & & 1.7 & 1.6 & 1.7 & 1.2 & 1.1 & 2.4 & 2.4 & 3.6 & 3.6 & 4.8 & 4.8 & 4.6 & 4.6 \\
\hline Social security tax & 3.4 & 3.6 & 2.1 & 1.9 & 2.0 & $\ldots$ & $\ldots$ & $\ldots$ & $\ldots$ & $\ldots$ & $\ldots$ & $\ldots$ & $\ldots$ & $\ldots$ & $\ldots$ \\
\hline Trade tax & 4.7 & 4.1 & $\ldots$ & 3.3 & 3.1 & 0.7 & 0.8 & 1.4 & 1.4 & 2.2 & 2.2 & 3.1 & 3.1 & 2.8 & 2.6 \\
\hline Excise tax & & & $\ldots$ & 4.9 & 9.3 & 1.0 & 1.3 & 2.1 & 2.1 & 3.3 & 3.3 & 4.7 & 4.7 & 4.7 & 4.5 \\
\hline Goods and services tax (GST) & 10.9 & 12.6 & $\ldots$ & 9.5 & 7.0 & 2.3 & 2.2 & 4.7 & 4.7 & 7.1 & 7.1 & 10.1 & 10.1 & 9.9 & 9.7 \\
\hline Business tax & 6.0 & 7.5 & 5.4 & 7.0 & 7.0 & 1.1 & 2.2 & 2.3 & 2.9 & 4.5 & 4.8 & 6.4 & 6.4 & 6.4 & 6.4 \\
\hline Other & 3.2 & 3.2 & 2.6 & 2.9 & 3.1 & 1.2 & 0.5 & 1.7 & 1.7 & 2.4 & 2.4 & 2.9 & 2.9 & 3.0 & 2.4 \\
\hline Pension Fund contribution & $\ldots$ & $\ldots$ & $\ldots$ & $\ldots$ & $\ldots$ & 0.1 & 0.1 & 0.2 & 0.2 & 0.3 & 0.3 & 0.4 & 0.4 & 0.6 & 0.6 \\
\hline Nontax & 4.7 & 4.4 & 3.8 & 4.3 & 4.2 & 0.6 & 1.2 & 1.6 & 1.8 & 3.1 & 3.3 & 4.0 & 4.2 & 3.8 & 3.8 \\
\hline Fees and charges & 1.7 & 1.7 & 1.7 & 1.6 & 1.7 & 0.4 & 0.4 & 0.8 & 0.8 & 1.2 & 1.2 & 1.7 & 1.7 & 1.7 & 1.7 \\
\hline Dividends from parastatals & 0.7 & 1.4 & 1.1 & 2.0 & 1.8 & 0.1 & 0.6 & 0.4 & 0.7 & 1.4 & 1.6 & 1.7 & 2.0 & 1.6 & 1.6 \\
\hline Other & 2.3 & 1.3 & 0.9 & 0.6 & 0.7 & 0.1 & 0.2 & 0.4 & 0.3 & 0.5 & 0.5 & 0.6 & 0.6 & 0.6 & 0.6 \\
\hline External grants & 3.7 & 4.4 & 3.1 & 1.5 & 0.9 & 0.8 & 0.0 & 2.0 & 2.2 & 3.1 & 3.2 & 4.0 & 4.0 & 1.6 & 0.9 \\
\hline Expenditure and net lending & 40.0 & 33.8 & 33.8 & 34.3 & 33.6 & 8.7 & 6.8 & 17.8 & 18.7 & 27.3 & 28.6 & 37.8 & 38.8 & 35.3 & 34.2 \\
\hline Current expenditure & 28.7 & 31.0 & 28.9 & 29.3 & 28.1 & 6.2 & 6.0 & 12.7 & 13.2 & 19.5 & 20.4 & 27.4 & 28.1 & 27.3 & 26.4 \\
\hline Primary current expenditure & 21.5 & 21.3 & 22.2 & 22.6 & 21.8 & 5.3 & 5.1 & 11.5 & 11.9 & 17.6 & 18.2 & 25.0 & 25.4 & 24.5 & 23.8 \\
\hline Wages and salaries & 7.9 & 7.1 & 6.6 & 6.1 & 6.1 & 1.9 & 1.7 & 3.8 & 3.8 & 5.7 & 5.7 & 7.6 & 7.5 & 7.5 & 7.5 \\
\hline Goods and services & 5.6 & 6.7 & 6.9 & 8.2 & 7.5 & 1.4 & 1.4 & 3.3 & 3.3 & 5.2 & 5.2 & 8.1 & 8.1 & 7.6 & 7.4 \\
\hline Transfers & 7.9 & 7.3 & 8.6 & 8.2 & 8.1 & 2.0 & 2.0 & 4.3 & 4.8 & 6.7 & 7.3 & 9.2 & 9.6 & 9.3 & 8.9 \\
\hline Other & 0.1 & 0.2 & 0.1 & 0.1 & 0.1 & 0.0 & 0.0 & 0.1 & 0.1 & 0.1 & 0.1 & 0.1 & 0.1 & 0.1 & 0.1 \\
\hline Interest payments due & 7.2 & 9.7 & 6.7 & 6.7 & 6.3 & 0.8 & 0.8 & 1.3 & 1.4 & 1.9 & 2.1 & 2.4 & 2.7 & 2.8 & 2.6 \\
\hline Foreign interest & 3.9 & 4.0 & 2.8 & 3.6 & 3.6 & 0.6 & 0.5 & 0.8 & 0.6 & 1.2 & 1.0 & 1.4 & 1.2 & 1.5 & 1.6 \\
\hline Domestic interest & 3.3 & 5.7 & 3.9 & 3.1 & 2.7 & 0.2 & 0.4 & 0.5 & 0.7 & 0.7 & 1.1 & 1.0 & 1.5 & 1.3 & 1.0 \\
\hline Capital expenditure $^{2}$ & 2.1 & 5.7 & 7.4 & 8.4 & 9.0 & 2.6 & 0.8 & 5.0 & 5.2 & 7.5 & 7.7 & 10.4 & 10.1 & 7.4 & 7.3 \\
\hline Net lending & 9.1 & -2.9 & -3.1 & -3.4 & -3.4 & 0.0 & 0.1 & 0.0 & 0.2 & 0.0 & 0.5 & 0.0 & 0.7 & 0.6 & 0.5 \\
\hline Primary balance & 3.9 & 15.7 & 7.4 & 9.4 & 9.1 & 1.2 & 3.5 & 2.0 & 2.2 & 4.3 & 3.8 & 5.0 & 4.5 & 5.0 & 4.0 \\
\hline Overall balance, commitment & -3.4 & 6.0 & 0.7 & 2.7 & 2.8 & 0.3 & 2.6 & 0.7 & 0.8 & 2.4 & 1.7 & 2.7 & 1.8 & 2.1 & 1.4 \\
\hline Change in arrears & 0.4 & 0.9 & -0.9 & -0.4 & 0.0 & 0.0 & -0.1 & 0.0 & -0.1 & 0.0 & -0.1 & 0.0 & -0.1 & 0.0 & 0.0 \\
\hline External interest & 1.9 & 3.0 & 0.0 & 0.5 & 0.8 & 0.0 & 0.0 & 0.0 & 0.0 & 0.0 & 0.0 & 0.0 & 0.0 & 0.0 & 0.0 \\
\hline Budget & -1.6 & -2.1 & -0.9 & -0.9 & -0.8 & 0.0 & -0.1 & 0.0 & -0.1 & 0.0 & -0.1 & 0.0 & -0.1 & 0.0 & 0.0 \\
\hline Overall balance, cash basis (after grants) & -3.0 & 6.9 & -0.2 & 2.3 & 2.8 & 0.3 & 2.6 & 0.7 & 0.7 & 2.4 & 1.6 & 2.7 & 1.7 & 2.1 & 1.4 \\
\hline Financing & 3.0 & -6.9 & 0.2 & -2.3 & -2.8 & -0.3 & -2.6 & -0.7 & -0.7 & -2.4 & -1.6 & -2.7 & -1.7 & -2.1 & -1.4 \\
\hline Foreign financing & 2.7 & -1.6 & 0.4 & 0.6 & 2.1 & 0.1 & -0.1 & 0.5 & 0.2 & 0.8 & 0.3 & 1.1 & 0.5 & -0.4 & -1.0 \\
\hline Disbursements & 7.7 & 1.3 & 3.9 & 4.3 & 5.2 & 0.6 & 0.0 & 1.2 & 0.6 & 1.8 & 1.2 & 2.4 & 1.8 & 0.8 & 0.6 \\
\hline Project loans & 7.7 & 1.3 & 1.1 & 1.4 & 2.2 & 0.6 & 0.0 & 1.2 & 0.6 & 1.8 & 1.2 & 2.4 & 1.8 & 0.8 & 0.6 \\
\hline Program/budget support & 0.0 & 1.1 & 2.9 & 2.9 & 3.0 & 0.0 & 0.0 & 0.0 & 0.0 & 0.0 & 0.0 & 0.0 & 0.0 & 0.0 & 0.0 \\
\hline Scheduled amortization & -18.7 & -10.2 & -3.7 & -4.9 & -4.9 & -0.5 & -0.3 & -0.7 & -0.6 & -1.0 & -1.0 & -1.4 & -1.5 & -1.3 & -1.6 \\
\hline Change in amortization arrears & 13.7 & -11.3 & -17.4 & -25.2 & -24.9 & 0.0 & 0.2 & 0.0 & 0.2 & 0.0 & 0.2 & 0.0 & 0.2 & 0.0 & 0.0 \\
\hline Clearance of arrears & 0.0 & 18.1 & 17.4 & 26.4 & 26.7 & 0.0 & 0.0 & 0.0 & 0.0 & 0.0 & 0.0 & 0.0 & 0.0 & 0.0 & 0.0 \\
\hline Debt service relief & 0.0 & 0.5 & 0.1 & 0.0 & 0.0 & 0.0 & 0.0 & 0.0 & 0.0 & 0.0 & 0.0 & 0.0 & 0.0 & 0.0 & 0.0 \\
\hline Domestic financing & -0.8 & -6.6 & -6.5 & -4.7 & -5.9 & -1.1 & -2.0 & -2.2 & -1.1 & -4.7 & -2.9 & -5.6 & -3.8 & -2.9 & -1.2 \\
\hline Bank financing & -1.1 & -7.7 & -4.4 & -2.5 & -2.1 & -1.0 & -1.6 & -1.9 & -0.8 & -4.2 & -2.4 & -5.0 & -3.3 & -2.6 & -1.1 \\
\hline CBS & $\ldots$ & -3.5 & -1.6 & -1.3 & -1.2 & -0.8 & -0.2 & -1.7 & -0.6 & -2.1 & -0.6 & -2.1 & -0.6 & 0.0 & 0.0 \\
\hline CBS recapitalization & $\ldots$ & $\ldots$ & 1.7 & 1.7 & 1.7 & $\ldots$ & $\ldots$ & $\ldots$ & $\ldots$ & $\ldots$ & $\ldots$ & $\ldots$ & $\ldots$ & $\cdots$ & $\ldots$ \\
\hline Commercial banks & $\ldots$ & -4.2 & -4.5 & -2.8 & -2.6 & -0.2 & -1.5 & -0.3 & -0.2 & -2.2 & -1.8 & -2.9 & -2.7 & -2.6 & -1.1 \\
\hline Nonbank & 0.3 & 1.1 & -0.5 & -2.2 & -3.8 & -0.1 & -0.4 & -0.2 & -0.3 & -0.5 & -0.5 & -0.6 & -0.6 & -0.3 & -0.1 \\
\hline Privatization and long-term lease of fixed assets & 2.5 & 2.1 & 1.4 & 1.4 & 1.4 & 0.3 & 0.2 & 0.3 & 0.5 & 0.3 & 0.8 & 1.2 & 1.2 & 1.2 & 0.8 \\
\hline Statistical discrepancy & -1.4 & 0.2 & 0.5 & 0.0 & -0.4 & 0.0 & -0.7 & 0.0 & -0.7 & 0.0 & -0.7 & 0.0 & -0.7 & 0.0 & 0.0 \\
\hline Fiscal financing gap & 0.0 & 0.0 & 4.5 & 0.4 & 0.0 & 0.3 & 0.0 & 0.3 & 0.3 & 0.6 & 0.8 & 0.6 & 1.1 & 0.0 & 0.0 \\
\hline Memorandum items: & & & & & & & & & & & & & & & \\
\hline Nominal GDP & 8,710 & 10,725 & 11,206 & 11,303 & 11,294 & 12,000 & 12,016 & 12,000 & 12,016 & 12,000 & 12,016 & 12,000 & 12,016 & 12,953 & 14,064 \\
\hline Public domestic debt (\% GDP) ${ }^{3}$ & 52.3 & 36.0 & 28.8 & 30.8 & 33.6 & 28.7 & 28.8 & 28.5 & 30.2 & 26.4 & 28.4 & 25.5 & 27.4 & 22.5 & 19.5 \\
\hline Publicly guaranteed domestic debt (\% GDP) & $\ldots$ & 1.6 & $\ldots$ & $\ldots$ & 2.2 & $\ldots$ & $\ldots$ & $\ldots$ & $\ldots$ & $\ldots$ & $\ldots$ & $\ldots$ & 2.6 & 2.0 & 1.6 \\
\hline
\end{tabular}




\begin{tabular}{|c|c|c|c|c|c|c|c|c|c|c|c|c|c|c|c|c|c|c|}
\hline & \multirow[t]{3}{*}{2008} & \multirow[t]{3}{*}{2009} & \multicolumn{8}{|c|}{2010} & \multicolumn{8}{|c|}{2011} \\
\hline & & & \multicolumn{2}{|c|}{ Mar. } & \multicolumn{2}{|c|}{ June } & \multicolumn{2}{|c|}{ Sep. } & \multicolumn{2}{|c|}{ Dec. } & \multicolumn{2}{|c|}{ Mar. } & \multicolumn{2}{|c|}{ June } & \multicolumn{2}{|c|}{ Sep. } & \multicolumn{2}{|c|}{ Dec. } \\
\hline & & & Prog. & Act. & Prog. & Act. & Prog. & Act. & Prog. & Prel. & Prog. & Proj. & Prog. & Proj. & Prog. & Proj. & Prog. & Proj. \\
\hline Monetary survey & \multicolumn{18}{|c|}{ (Seychelles rupees millions, unless otherwise indicated) } \\
\hline Net foreign assets & 2,105 & 2,897 & 2,388 & 3,345 & 3,079 & 3,256 & 3,244 & 3,641 & 4,002 & 3,644 & 4,183 & 3,984 & 4,413 & 3,941 & 4,796 & 4,075 & 4,954 & 4,098 \\
\hline Central bank & 1,050 & 1,998 & 1,802 & 2,397 & 2,140 & 2,230 & 2,304 & 2,357 & 2,560 & 2,709 & 2,624 & 2,788 & 2,688 & 2,781 & 2,834 & 2,821 & 2,994 & 2,786 \\
\hline Deposit money banks & 1,055 & 899 & 586 & 947 & 939 & 1,025 & 941 & 1,284 & 1,442 & 935 & 1,559 & 1,195 & 1,725 & 1,160 & 1,961 & 1,254 & 1,960 & 1,313 \\
\hline Net domestic assets & 3,879 & 3,504 & 3,834 & 3,223 & 3,684 & 3,281 & 3,952 & 3,222 & 3,197 & 3,631 & 3,223 & 3,436 & 3,230 & 3,641 & 3,062 & 3,550 & 3,046 & 3,580 \\
\hline Domestic credit & 5,949 & 4,796 & 4,518 & 4,811 & 5,028 & 4,906 & 5,018 & 5,320 & 5,362 & 5,714 & 5,300 & 5,754 & 5,300 & 5,912 & 5,126 & 5,754 & 5,112 & 5,700 \\
\hline Net claims on the government & 3,082 & 2,255 & 1,707 & 2,115 & 2,216 & 2,158 & 2,082 & 2,474 & 2,277 & 2,630 & 2,159 & 2,443 & 2,043 & 2,542 & 1,769 & 2,350 & 1,678 & 2,247 \\
\hline Credit to the economy & 2,867 & 2,540 & 2,811 & 2,695 & 2,813 & 2,749 & 2,936 & 2,846 & 3,085 & 3,085 & 3,142 & 3,311 & 3,256 & 3,369 & 3,358 & 3,404 & 3,434 & 3,453 \\
\hline Other items, net & $-2,071$ & $-1,292$ & -684 & $-1,587$ & $-1,344$ & $-1,625$ & $-1,066$ & $-2,097$ & $-2,165$ & $-2,083$ & $-2,078$ & $-2,318$ & $-2,069$ & $-2,271$ & $-2,065$ & $-2,204$ & $-2,066$ & $-2,121$ \\
\hline Broad money & 5,983 & 6,401 & 6,222 & 6,568 & 6,763 & 6,537 & 7,197 & 6,864 & 7,199 & 7,275 & 7,406 & 7,420 & 7,643 & 7,581 & 7,857 & 7,625 & 8,000 & 7,678 \\
\hline Currency in circulation & 478 & 500 & 509 & 497 & 519 & 507 & 549 & 512 & 562 & 580 & 608 & 588 & 614 & 590 & 621 & 602 & 628 & 604 \\
\hline Foreign currency deposits & 1,913 & 1,610 & 1,600 & 1,682 & 1,655 & 1,616 & 1,762 & 1,828 & 1,759 & 1,708 & 1,802 & 2,011 & 1,863 & 2,027 & 1,918 & 2,037 & 1,954 & 2,051 \\
\hline Local currency deposits & 3,593 & 4,291 & 4,113 & 4,388 & 4,589 & 4,414 & 4,886 & 4,524 & 4,878 & 4,987 & 4,997 & 4,821 & 5,166 & 4,964 & 5,318 & 4,987 & 5,419 & 5,023 \\
\hline \multicolumn{19}{|l|}{ Central bank } \\
\hline Net foreign assets & 1,050 & 1,998 & 1,802 & 2,397 & 2,140 & 2,230 & 2,304 & 2,357 & 2,560 & 2,709 & 2,624 & 2,788 & 2,688 & 2,781 & 2,834 & 2,821 & 2,994 & 2,786 \\
\hline Foreign assets & 1,207 & 2,207 & 2,044 & 2,648 & 2,468 & 2,490 & 2,675 & 2,664 & 2,921 & 3,087 & 2,987 & 3,177 & 3,052 & 3,242 & 3,200 & 3,289 & 3,463 & 3,316 \\
\hline Of which: official reserves & 843 & 1,907 & 1,740 & 2,470 & 2,267 & 2,273 & 2,473 & 2,499 & 2,671 & 2,888 & 2,723 & 2,986 & 2,775 & 2,995 & 2,909 & 3,033 & 3,158 & 3,055 \\
\hline Foreign liabilities & 157 & 210 & 242 & 250 & 329 & 259 & 372 & 307 & 361 & 379 & 363 & 389 & 364 & 461 & 365 & 467 & 468 & 530 \\
\hline Net domestic assets & 70 & -702 & -266 & -973 & -548 & -686 & -630 & -765 & -804 & -962 & -837 & $-1,016$ & -881 & $-1,026$ & $-1,007$ & $-1,064$ & $-1,149$ & $-1,021$ \\
\hline Domestic credit & 851 & -657 & -279 & -779 & -295 & -420 & -302 & -385 & -409 & -506 & -525 & -486 & -575 & -492 & -705 & -484 & -845 & -413 \\
\hline Government (net) $^{1}$ & 1,099 & 724 & 55 & 547 & 609 & 614 & 672 & 761 & 761 & 780 & 661 & 762 & 561 & 712 & 511 & 712 & 511 & 712 \\
\hline Commercial banks & -22 & $-1,266$ & -212 & $-1,202$ & -781 & -910 & -851 & -860 & -884 & $-1,135$ & -900 & $-1,079$ & -850 & $-1,035$ & -930 & $-1,027$ & $-1,070$ & -955 \\
\hline Other (parastatals) & -226 & -114 & -122 & -123 & -123 & -123 & -123 & -286 & -286 & -151 & -286 & -170 & -286 & -170 & -286 & -170 & -286 & -170 \\
\hline Other items, net & -780 & -45 & 13 & -195 & -254 & -267 & -328 & -380 & -395 & -456 & -313 & -530 & -307 & -534 & -302 & -580 & -304 & -608 \\
\hline Reserve money & 1,120 & 1,296 & 1,536 & 1,424 & 1,591 & 1,544 & 1,674 & 1,592 & 1,756 & 1,746 & 1,787 & 1,772 & 1,807 & 1,755 & 1,827 & 1,757 & 1,846 & 1,765 \\
\hline Currency in circulation & 478 & 500 & 509 & 497 & 519 & 507 & 549 & 512 & 562 & 580 & 608 & 588 & 614 & 590 & 621 & 602 & 628 & 604 \\
\hline Commercial bank reserves (includes cash in vault) & 642 & 796 & 1,028 & 927 & 1,072 & 1,037 & 1,125 & 1,080 & 1,194 & 1,166 & 1,179 & 1,184 & 1,193 & 1,165 & 1,206 & 1,155 & 1,218 & 1,161 \\
\hline Of which: required reserves in foreign currency ${ }^{2,3}$ & & 170 & 161 & 176 & 188 & 181 & 200 & 195 & 213 & 193 & 225 & 215 & 238 & 264 & 250 & 265 & 263 & 267 \\
\hline required reserves in domestic currency ${ }^{3}$ & 745 & 467 & 411 & 489 & 473 & 508 & 502 & 509 & 498 & 545 & 509 & 565 & 526 & 657 & 542 & 660 & 552 & 665 \\
\hline \multicolumn{19}{|l|}{ Memorandum items: } \\
\hline Gross international reserves (US $\$$ millions) ${ }^{4}$ & 51 & 169 & 158 & 210 & 192 & 182 & 207 & 202 & 218 & 238 & 221 & 243 & 225 & 241 & 235 & 240 & 253 & 241 \\
\hline Foreign currency deposits (US\$ millions) & 115 & 143 & 145 & 143 & 140 & 130 & 147 & 148 & 144 & 141 & 146 & 164 & 151 & 163 & 155 & 161 & 157 & 162 \\
\hline Broad money growth (12-month percent change) & 29.0 & 7.0 & 15.0 & 21.4 & 33.5 & 24.6 & 33.0 & 26.9 & 12.5 & 13.7 & 12.8 & 13.0 & 16.9 & 16.0 & 14.5 & 11.1 & 11.1 & 5.5 \\
\hline Credit to the economy (12-month percent change) & 48.6 & -11.4 & -2.7 & 2.9 & 14.3 & 11.7 & 19.1 & 15.4 & 21.5 & 21.4 & 16.6 & 22.9 & 18.5 & 22.6 & 18.0 & 19.6 & 11.3 & 11.9 \\
\hline Reserve money (12-month percent change) & 0.6 & 15.7 & 46.2 & 35.5 & 42.2 & 38.0 & 43.1 & 36.1 & 35.5 & 34.7 & 25.5 & 24.5 & 17.0 & 13.7 & 14.6 & 10.4 & 5.1 & 1.1 \\
\hline Money multiplier (broad money/reserve money) & 5.3 & 4.9 & 4.1 & 4.6 & 4.3 & 4.2 & 4.3 & 4.3 & 4.1 & 4.2 & 4.1 & 4.2 & 4.2 & 4.3 & 4.3 & 4.3 & 4.3 & 4.4 \\
\hline Velocity (GDP/broad money; end of period) & 1.5 & 1.7 & 1.5 & 1.6 & 1.5 & 1.6 & 1.5 & 1.6 & 1.6 & 1.6 & 1.5 & 1.6 & 1.5 & 1.6 & 1.5 & 1.6 & 1.5 & 1.6 \\
\hline Change in net credit to government (change in quarter) & -158 & 148 & -15 & -140 & 100 & 42 & -133 & 317 & -197 & 155 & 397 & -187 & -234 & 100 & -390 & -192 & -366 & -103 \\
\hline
\end{tabular}

Sources: Central Bank of Seychelles and IMF staff estimates and projections.

${ }^{1}$ Assumes recapitalization of the CBS by SR 188 million in three equal quarterly installments in 2010 .

${ }^{2}$ Reserve requirements on foreign currency deposits were introduced in 2009.

${ }^{3}$ Reserve requirements were lowered from 13\% to $10 \%$ in 2009, but raised back to $13 \%$ in April 2011.

${ }^{4}$ Reflects an increase in SDR holdings following SDR allocation of SDR 7.9 million in 2009. 
Table 5. Seychelles: Financial Soundness Indicators for the Banking Sector, 2007-10 ${ }^{1}$

(Percent, end of period)

\begin{tabular}{|c|c|c|c|c|c|c|c|c|c|c|c|c|c|c|c|c|}
\hline & \multicolumn{4}{|c|}{2007} & \multicolumn{4}{|c|}{2008} & \multicolumn{4}{|c|}{2009} & \multicolumn{4}{|c|}{2010} \\
\hline & Q1 & Q2 & Q3 & Q4 & Q1 & Q2 & Q3 & $\mathrm{Q} 4$ & Q1 & Q2 & Q3 & Q4 & Q1 & Q2 & Q3 & Q4 \\
\hline \multicolumn{17}{|l|}{ Capital adequacy } \\
\hline Regulatory capital to risk weighted assets & 20.4 & 21.0 & 18.2 & 18.2 & 18.4 & 17.6 & 17.9 & 19.4 & 13.6 & 17.8 & 22.0 & 21.7 & 21.4 & 22.1 & 21.7 & 21.5 \\
\hline Regulatory tier 1 capital to risk weighted assets & 14.8 & 16.1 & 14.8 & 12.9 & 12.9 & 14.0 & 13.4 & 10.4 & 12.9 & 17.1 & 21.3 & 21.0 & 20.7 & 21.5 & 21.1 & 20.8 \\
\hline Capital to assets (net worth) & 6.2 & 6.5 & 6.4 & 6.4 & 6.9 & 6.6 & 7.0 & 8.4 & 9.6 & 8.7 & 9.7 & 9.9 & 9.3 & 8.7 & 9.1 & 9.2 \\
\hline Net tangible capitalization ${ }^{2}$ & 6.2 & 6.6 & 6.5 & 6.5 & 7.0 & 6.6 & 7.1 & 8.4 & 9.6 & 8.7 & 9.8 & 9.9 & 9.4 & 8.8 & 9.1 & 9.3 \\
\hline \multicolumn{17}{|l|}{ Asset quality } \\
\hline Foreign exchange loans to total loans & 24.1 & 25.1 & 28.7 & 30.8 & 32.7 & 32.9 & 31.6 & 50.8 & 50.5 & 43.0 & 41.1 & 37.2 & 34.3 & 29.7 & 27.8 & 25.5 \\
\hline Nonperforming loans to gross loans & 3.7 & 2.9 & 2.5 & 2.3 & 2.0 & 2.1 & 2.0 & 2.0 & 2.3 & 4.3 & 4.2 & 3.8 & 4.1 & 6.9 & 6.4 & 5.5 \\
\hline Provisions as percentage of nonperforming loans & 38.2 & 44.1 & 45.6 & 45.9 & 51.2 & 50.3 & 59.6 & 58.3 & 56.8 & 35.2 & 32.6 & 33.8 & 41.7 & 26.0 & 26.0 & 31.4 \\
\hline \multicolumn{17}{|l|}{ Earnings and profitability } \\
\hline Return on assets (annualized) & 3.9 & 3.4 & 5.0 & 6.6 & 4.3 & 3.4 & 2.8 & 16.1 & 5.3 & 3.0 & -0.2 & 3.2 & 3.4 & 5.0 & 3.0 & 3.7 \\
\hline Return on equity (annualized) & 59.1 & 50.4 & 68.3 & 93.0 & 59.5 & 47.9 & 39.3 & 199.4 & 55.1 & 35.4 & -2.8 & 32.4 & 33.6 & 54.3 & 33.7 & 40.0 \\
\hline Interest margin to gross income & 60.0 & 66.1 & 57.9 & 46.0 & 58.7 & 62.8 & 58.3 & 24.2 & 62.1 & 83.7 & 105.5 & 68.6 & 56.5 & 60.2 & 59.9 & 50.6 \\
\hline Noninterest expense to gross income & 35.5 & 39.7 & 33.3 & 32.3 & 33.2 & 43.5 & 49.4 & 17.8 & 36.4 & 48.7 & 116.5 & 49.1 & 44.0 & 52.0 & 47.6 & 39.9 \\
\hline Net interest margin (annualized) $)^{3}$ & 3.6 & 3.7 & 4.3 & 4.5 & 3.8 & 3.8 & 3.1 & 4.8 & 5.1 & 4.8 & 4.2 & 4.0 & 3.1 & 3.6 & 3.4 & 3.1 \\
\hline Net noninterest margin (annualized) ${ }^{4}$ & 0.3 & -0.3 & 0.6 & 2.1 & 0.5 & -0.4 & -0.4 & 11.4 & 0.1 & -1.9 & -4.9 & -1.0 & 0.0 & -0.7 & -0.4 & 0.6 \\
\hline Expense to income & 48.5 & 53.5 & 44.8 & 42.2 & 46.6 & 55.9 & 62.5 & 25.5 & 52.3 & 62.2 & 111.2 & 58.0 & 53.0 & 58.0 & 53.6 & 46.6 \\
\hline Interest expense to gross income & 25.2 & 29.5 & 20.8 & 17.2 & 25.0 & 28.0 & 35.0 & 10.2 & 33.2 & 35.8 & 47.1 & 21.2 & 19.0 & 14.2 & 12.8 & 12.5 \\
\hline \multicolumn{17}{|l|}{ Liquidity } \\
\hline Core liquid assets to total assets ${ }^{5}$ & 19.2 & 18.6 & 11.4 & 10.0 & 30.8 & 34.6 & 36.4 & 37.8 & 36.0 & 35.0 & 37.7 & 43.2 & 43.5 & 44.7 & 44.7 & 46.9 \\
\hline Broad liquid assets to total assets ${ }^{6}$ & 54.8 & 55.8 & 44.3 & 39.4 & 59.2 & 60.9 & 60.0 & 55.7 & 50.8 & 53.0 & 57.6 & 58.4 & 58.6 & 60.0 & 58.1 & 58.9 \\
\hline Liquid assets (broad) to short term liabilities & 57.3 & 57.5 & 46.7 & 40.8 & 59.3 & 61.6 & 87.1 & 56.5 & 53.2 & 56.2 & 62.1 & 62.7 & 65.0 & 63.4 & 62.1 & 62.9 \\
\hline Liquid assets (broad) to total liabilities & 58.7 & 60.1 & 47.8 & 42.3 & 63.9 & 65.5 & 64.8 & 61.2 & 56.2 & 58.0 & 63.8 & 64.7 & 64.7 & 65.7 & 63.8 & 64.9 \\
\hline Liquid assets to deposit liabilities & 64.0 & 64.5 & 52.5 & 46.0 & 68.3 & 72.4 & 71.7 & 66.4 & 63.1 & 63.8 & 71.4 & 69.4 & 72.4 & 72.0 & 69.1 & 69.1 \\
\hline \multicolumn{17}{|l|}{ Foreign exchange exposure } \\
\hline Net open foreign exchange position to capital & 2.6 & 14.1 & 28.3 & 28.5 & 24.9 & 31.3 & 21.3 & 58.9 & 44.4 & 32.4 & 21.9 & 33.1 & 22.2 & 24.7 & 29.2 & 5.6 \\
\hline
\end{tabular}

Source: Central Bank of Seychelles.

${ }^{1}$ Excluding purely offshore banks.

${ }^{2}$ Defined as: equity capital/(assets-interest in suspense-provisions).

${ }^{3}$ Defined as: (Interest income - interest expense)/average assets.

${ }^{4}$ Defined as: (Noninterest income - noninterest expense)/average assets

${ }^{5}$ Core liquid assets include cash, balances with CBS and deposits with other banks.

${ }^{6}$ Broad liquid assets include core liquid assets plus investments in government securities. 
Table 6. Seychelles: Fund Disbursements and Timing of Reviews Under the Proposed Three-Year EFF, 2009-12

\begin{tabular}{|c|c|c|c|c|}
\hline \multirow{2}{*}{$\begin{array}{l}\text { Program } \\
\text { Review }\end{array}$} & \multirow[b]{2}{*}{ Date of availability } & \multirow[b]{2}{*}{ Conditions } & \multirow{2}{*}{\multicolumn{2}{|c|}{$\frac{\text { Amount }}{\text { (millions of SDR) (percent of quota) }}$}} \\
\hline & & & & \\
\hline & December 22, 2009 & Board approval of the EFF arrangement. & 0.88 & 10 \\
\hline & March 15, 2010 & $\begin{array}{l}\text { Compliance with end-December } 2009 \\
\text { quantitative performance criteria and } \\
\text { completion of a financing assurances } \\
\text { review (if needed) }\end{array}$ & 2.20 & 25 \\
\hline \multirow[t]{2}{*}{ First } & June 15, 2010 & $\begin{array}{l}\text { Completion of first program review and } \\
\text { financing assurances review (if needed) } \\
\text { and compliance with end-March } 2010 \\
\text { quantitative performance criteria. }\end{array}$ & 2.20 & 25 \\
\hline & September 15, 2010 & $\begin{array}{l}\text { Compliance with end-June } 2010 \\
\text { quantitative performance criteria and } \\
\text { completion of a financing assurances } \\
\text { review (if needed) }\end{array}$ & 2.20 & 25 \\
\hline Second & December 15, 2010 & $\begin{array}{l}\text { Completion of second program review } \\
\text { and financing assurances review (if } \\
\text { needed) and compliance with end- } \\
\text { September } 2010 \text { quantitative } \\
\text { performance criteria. }\end{array}$ & 1.76 & 20 \\
\hline Third & March 31, 2011 & $\begin{array}{l}\text { Compliance with end-December } 2010 \\
\text { quantitative performance criteria and } \\
\text { completion of a financing assurances } \\
\text { review (if needed). }\end{array}$ & 3.52 & 40 \\
\hline Fourth & September 30, 2011 & $\begin{array}{l}\text { Compliance with end-June } 2011 \\
\text { quantitative performance criteria and } \\
\text { completion of a financing assurances } \\
\text { review (if needed) }\end{array}$ & 3.08 & 35 \\
\hline Fifth & March 31, 2012 & $\begin{array}{l}\text { Compliance with end-December } 2011 \\
\text { quantitative performance criteria and } \\
\text { completion of a financing assurances } \\
\text { review (if needed) }\end{array}$ & 2.64 & 30 \\
\hline Sixth & September 30, 2012 & $\begin{array}{l}\text { Compliance with end-June } 2012 \\
\text { quantitative performance criteria and } \\
\text { completion of a financing assurances } \\
\text { review (if needed) }\end{array}$ & 1.32 & 15 \\
\hline Total & & & 19.8 & 225 \\
\hline
\end{tabular}


Table 7. Seychelles: Indicators of Fund Credit, 2008-15 (Millions of SDR)

\begin{tabular}{|c|c|c|c|c|c|c|c|c|}
\hline & 2008 & 2009 & 2010 & 2011 & 2012 & 2013 & 2014 & 2015 \\
\hline & \multicolumn{8}{|c|}{ Projections } \\
\hline \multicolumn{9}{|l|}{ Existing Fund credit } \\
\hline Stock ${ }^{1}$ & 6.2 & 11.9 & 20.2 & 20.2 & 16.8 & 11.3 & 8.9 & 7.4 \\
\hline Obligation & 0.0 & 0.0 & 0.0 & 0.2 & 3.7 & 5.7 & 2.6 & 1.7 \\
\hline Disbursements & 6.2 & 5.7 & 8.4 & 0.0 & 0.0 & 0.0 & 0.0 & 0.0 \\
\hline \multicolumn{9}{|l|}{ Prospective purchases under the EFF } \\
\hline Disbursements & & 0.0 & 0.0 & 6.6 & 4.0 & 0.0 & 0.0 & 0.0 \\
\hline Stock ${ }^{1}$ & & 0.0 & 0.0 & 6.6 & 10.6 & 10.6 & 10.6 & 10.3 \\
\hline Obligations $^{2}$ & & 0.0 & 0.0 & 0.1 & 0.1 & 0.2 & 0.2 & 0.5 \\
\hline Principal (repayments/repurchases) & & 0.0 & 0.0 & 0.0 & 0.0 & 0.0 & 0.0 & 0.3 \\
\hline Charges and interest & & 0.0 & 0.0 & 0.1 & 0.1 & 0.2 & 0.2 & 0.2 \\
\hline Stock of existing and prospective Fund credit 1 & 6.2 & 11.9 & 20.2 & 26.8 & 27.4 & 21.9 & 19.5 & 17.6 \\
\hline In percent of quota & 70.0 & 135.0 & 230.0 & 305.0 & 311.3 & 248.8 & 221.3 & 200.4 \\
\hline In percent of GDP & 1.0 & 2.4 & 3.3 & 4.4 & 4.2 & 3.2 & 2.6 & 2.2 \\
\hline In percent of exports of goods and services & 0.9 & 2.3 & 3.6 & 4.2 & 4.1 & 3.1 & 2.6 & 2.2 \\
\hline In percent of gross reserves & 18.4 & 11.1 & 13.1 & 17.5 & 17.1 & 12.5 & 9.9 & 7.4 \\
\hline \multicolumn{9}{|c|}{ Obligations to the Fund from existing and prospective } \\
\hline Fund arrangements & 0.0 & 0.0 & 0.0 & 0.3 & 3.8 & 5.9 & 2.7 & 2.1 \\
\hline In percent of quota & 0.3 & 0.3 & 0.1 & 3.2 & 43.4 & 66.7 & 31.1 & 24.1 \\
\hline In percent of GDP & 0.0 & 0.0 & 0.0 & 0.0 & 0.6 & 0.9 & 0.4 & 0.3 \\
\hline In percent of exports of goods and services & 0.0 & 0.0 & 0.0 & 0.0 & 0.6 & 0.8 & 0.4 & 0.3 \\
\hline In percent of gross reserves & 0.1 & 0.0 & 0.0 & 0.2 & 2.4 & 3.4 & 1.4 & 0.9 \\
\hline
\end{tabular}

Sources: IMF Finance Department; and IMF staff estimates and projections.

${ }^{1}$ End of period.

${ }^{2}$ Repayment schedule based on repurchase obligations. Obligations to the Fund from Existing and Prospective Fund Arrangements includes charges. 


\section{ANNEX I: ASSESSING THE ADEQUACY OF SEYCHELLES'S GROSS INTERNATIONAL RESERVES $^{4}$}

Despite the significant buildup over the past two years, Seychelles reserves remain slightly below the adequate levels suggested by various approaches. Reserves also appear to be lower compared to the peer group of small island economies.

This analysis focuses on adequacy of reserves based on a country's precautionary needs to absorb external shocks and avoid disruptive adjustments in domestic demand. ${ }^{5}$ The following approaches have been used:

- Traditional metrics include reserve coverage ratios in terms of months of imports (3 months), short-term debt (100 percent), and broad money (20 percent).

- Combination metrics include full coverage of short-term debt plus the current account deficit; and a Lipschitz, et al metric ${ }^{6}$, which uses reserve coverage of 20 percent of imports, 100 percent of short-term debt and 10 percent of broad money.

- Shock Scenarios estimate the potential needs for reserves in a stress test scenarios that takes into account country vulnerabilities.

- The revealed preferences approach estimates demand for reserves using a sample of 69 emerging market economies.

- Optimal reserves models estimate reserve levels based on a trade-off between opportunity costs and benefits in preventing and mitigating external shocks.

The results are summarized below:

\begin{tabular}{|c|c|c|c|}
\hline \multicolumn{4}{|c|}{ Reserves in months of imports } \\
\hline Traditional adequacy metrics ${ }^{1}$ & 0.8 & - & 3.0 \\
\hline Combination rules ${ }^{2}$ & 2.4 & - & 13.7 \\
\hline Shock scenarios & 4.5 & - & 4.9 \\
\hline Cross-country revealed preferences & 2.0 & - & 2.0 \\
\hline Optimality models & 2.6 & - & 3.6 \\
\hline Average (excl. extremes) & 2.9 & - & 3.4 \\
\hline \multicolumn{4}{|l|}{ Note: } \\
\hline \multicolumn{4}{|c|}{${ }^{1} 0.8$ reflects effect of debt rule converted to imports. } \\
\hline \multicolumn{4}{|c|}{${ }^{2}$ The maximum reflects the CA deficit effect. } \\
\hline
\end{tabular}

These estimates imply that on average an adequate level of reserves for Seychelles over the medium term is about 2.9-3.4 months of prospective imports. The projected increase in the stock of reserves from 2.2 months of prospective imports at end-2010 to 2.5 months in 2012 and 3.4 months in 2015 appear to be broadly in line with the above findings.

\footnotetext{
${ }^{4}$ Prepared by Nkunde Mwase. Details and calculations can be made available on request. The assessment does not cover the metrics recently developed by the Fund (International Monetary Fund, 2011, "Assessing Reserve Adequacy", International Monetary Fund) because of lack of data on other portfolio liabilities. ${ }^{5}$ Reserves could be held for other motives (e.g., intergenerational savings and export-promotion).

${ }^{6}$ Lipschitz, L., Miguel, M., and Alexandros, M., 2006, "Reserve Adequacy: Much Higher than You Thought?" mimeograph (Washington: International Monetary Fund).
} 
Seychelles reserves are low relative to other small tourism-island economies.
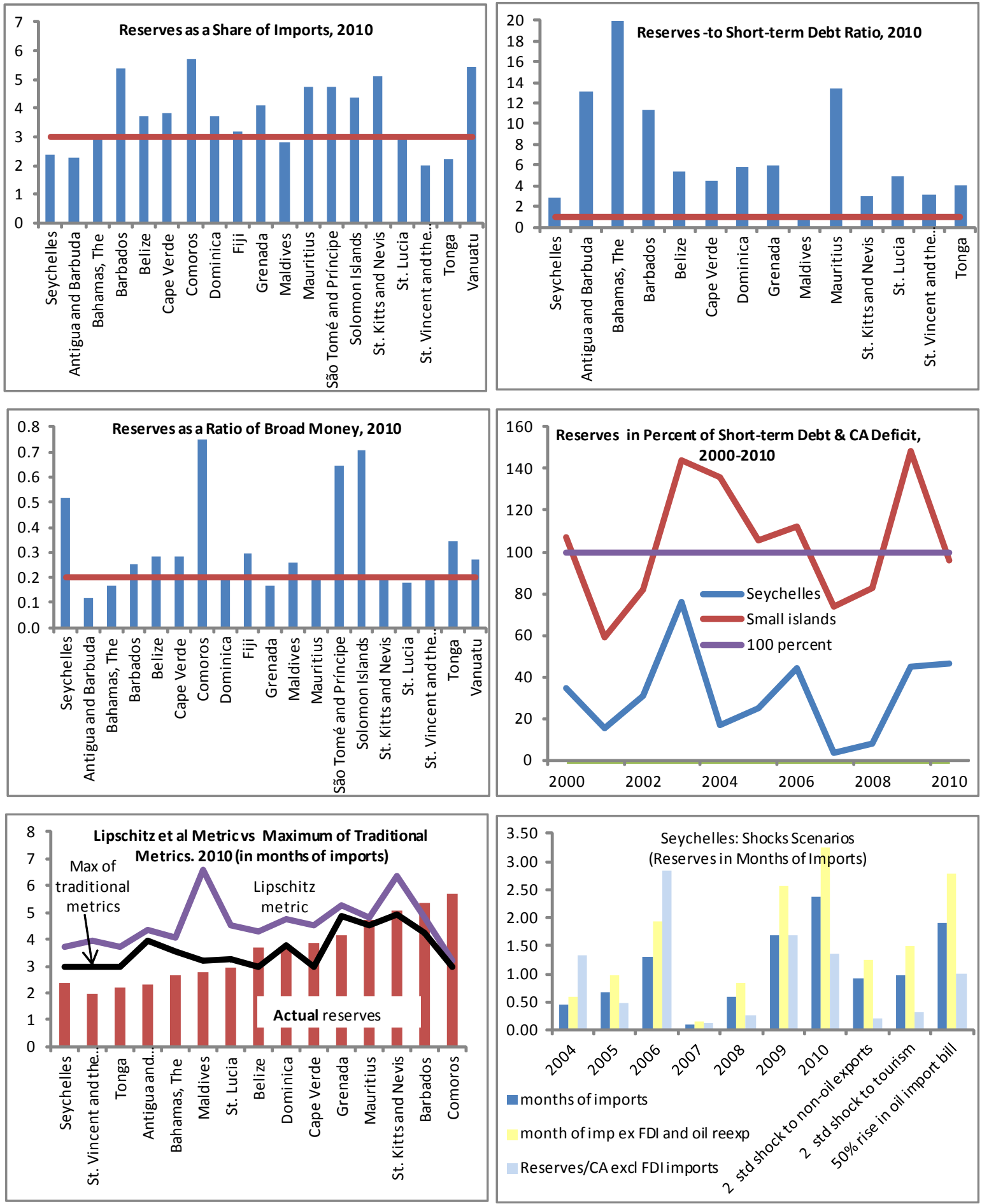

Sources: Seychelles authorities and IMF staff estimates. 


\section{Annex II. Transitioning to the GFSM 2001 Fiscal Presentation}

In March 2010, the IMF Executive Board called upon member countries to present fiscal data according to the Government Finance Statistics Manual 2001 (GFSM 2001) framework. ${ }^{7}$ A STA mission assisted the Ministry of Finance to develop a GFSM 2001-based reporting framework for post-program surveillance purposes. ${ }^{8}$ This appendix presents some preliminary results and highlights ongoing tasks that will be necessary to compile and begin reporting GFSM 2001-based data. This appendix does not impact the presentation of fiscal data used elsewhere in the report.

The STA mission found that there are adequate source data to establish a GFS presentation for Seychelles which would cover the operations of the consolidated central/general government (defined as the Budgetary Central Government and the Social Security Fund) as well as provide a monthly financial balance sheet of government that reconciles stocks and flows. Preliminary estimates indicated that the reporting of fiscal data on the basis of GFSM 2001 does not appear to substantially change the overall primary balance (including grants). The attendant reclassification of some specific transactions (in particular, "net lending" and some components of "privatization") and the recording of debt forgiveness as capital transfers would result in an increase of the primary balance from 9 to 10.5 percent of GDP in $2010 .^{9}$

Moving to a GFSM 2001-based reporting of central government operations will require:

- A thorough review of the classification, recording, and consolidation of transactions central/general government sector entities, with particular emphasis on data pertaining to transfers (grants), interest received from or paid to other general government units, and transactions related to social protection.

- The identification of any remaining off-budget government funds, following the incorporation of several central government units in the 2011 budget, reviewing their accounting practices, to find an appropriate solution that gives a transparent overview of their financial activities and fiscal impact.

\footnotetext{
${ }^{7}$ The mandate requires that a GFSM 2001-based Statement of Government Operations along with a financial balance sheet be included in staff reports as of May 2011, except for countries benefiting from Fund facilities. See decision No. 14565 outlined in Government Finance Statistics to Strengthen Fiscal Analysis (http://www.imf.org/external/pp/longres.aspx?id=4431)

${ }^{8}$ This work results from Seychelles participation in Phase II (July 2007 to May 2009) of the Government Finance Statistics module of the financed technical assistance program The Phase II GFS Module was part of the United Kingdom Department for International Development (DFID) funded project on Statistical Modules for Anglophone Africa in which five countries participated (Ethiopia, Ghana, Seychelles, Tanzania, Uganda).

${ }^{9}$ The STA mission helped the Ministry of Finance take the necessary steps to: (i) institutionalize a system of central government financial reporting that features GFS 2001 fiscal notions (including the Net Operating Balance, Net Lending/Borrowing, and Net Worth/Net Financial Worth); and (ii) develop and use balance sheets that reconcile opening and closing stock positions with transactions and other economic flows.. A baseline net financial worth of government was established, which will lay the foundation for quickly developing an overall estimate of government's net worth. The resulting financial balance sheet for 2010 is illustrated below.
} 
The next steps to further enhance the fiscal presentation include:

- A review of the derivation of the stock of currency and deposits as an asset of the budgetary central government.

- $\quad$ Ensuring that the completeness of information on stocks, transactions, and other economic flows not currently recorded in the financial assets and liabilities accounts of the budgetary central government (to better support the calculation of net financial worth and monitor its evolution).

- The introduction of a classification structure for nonfinancial assets aligned with the GFSM 2001 or the 2008 System of National Accounts into the budgetary central government's chart of accounts. This represents a deficiency that currently prohibits calculating the overall net worth of the central government.

The authorities will start reporting a monthly GFSM 2001-based fiscal presentation to the Fund for review by September 2011. The presentation will integrate stocks and flows, be compiled with monthly periodicity, and be reported-initially-within 30 days of the reference month, gradually improving the timeliness to no more than 10 days by end2012. The year-end 2008 central government balance sheet will be used as a benchmark.

TRANSACTIONS AFFECTING NET WORTH:

\begin{tabular}{|lr|}
\hline Revenue & $\mathbf{4 , 4 3 3 . 7}$ \\
Taxes & $3,294.6$ \\
Social contributions & 228.3 \\
Grants & 96.1 \\
Other revenue & 814.6 \\
Expense & $\mathbf{3 , 1 6 7 . 1}$ \\
Compensation of employees & 692.6 \\
Use of goods and services & 808.8 \\
Consumption of fixed capital & 0.0 \\
Interest & 711.4 \\
Subsidies & 271.0 \\
Grants & 0.0 \\
Social benefits & 671.4 \\
Other expense & 11.9 \\
Primary Operating Balance & $\mathbf{1 , 9 7 7 . 9}$ \\
Gross operating balance & $\mathbf{1 , 2 6 6 . 5}$ \\
Net operating balance & $\mathbf{1 , 2 6 6 . 5}$ \\
\hline
\end{tabular}

TRANSACTIONS IN NONFINANCIAL ASSETS:

Net Acquisition of Nonfinancial Assets

Fixed assets

Change in inventories

Valuables

Nonproduced assets

Net lending / borrowing

Overall Primary Balance

Including grants

In percent of GDP

Excluding grants

In percent of GDP

Net acquisition of financial assets

Currency and deposits

Securities other than shares

Loans

Shares and other equity

Insurance technical reserves

Financial derivatives

Other accounts receivable

Monetary gold and SDRs

Net incurrence of liabilities

Special Drawing Rights

Currency and deposits

Securities other than shares

Loans

Shares and other equity

Insurance technical reserves

Financial derivatives

Other accounts payable

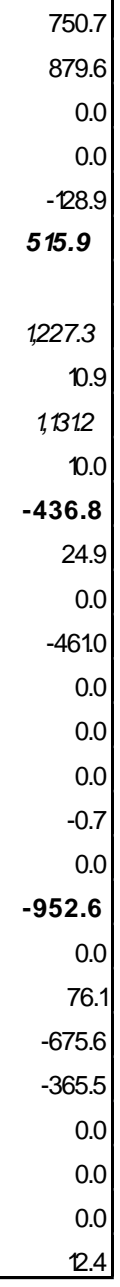




\begin{tabular}{|c|c|c|c|c|c|c|}
\hline \multirow[b]{2}{*}{ INTEGRATED BALANCE SHEET } & \multirow[b]{2}{*}{$\begin{array}{l}\text { Closing } \\
\text { balance } \\
12 / 2009\end{array}$} & \multirow[b]{2}{*}{ Transactions } & \multicolumn{2}{|c|}{ Other economic flows } & \multirow[b]{2}{*}{ Residual } & \multirow[b]{2}{*}{$\begin{array}{l}\text { Closing } \\
\text { balance } \\
12 / 2010\end{array}$} \\
\hline & & & \begin{tabular}{|c|} 
Holding \\
Gains and \\
Losses \\
\end{tabular} & $\begin{array}{c}\text { Other } \\
\text { volume } \\
\text { changes }\end{array}$ & & \\
\hline Net Financial Worth: & $\underline{-9,668}$ & $\underline{516}$ & $\underline{0}$ & $\underline{0}$ & $\underline{1,901}$ & $\underline{-7,252}$ \\
\hline Financial assets & 1,993 & -437 & 0 & 0 & 44 & 1,600 \\
\hline Currency and deposits & 762 & 25 & 0 & 0 & 18 & 805 \\
\hline Securities other than shares & 0 & 0 & 0 & 0 & 0 & 0 \\
\hline Loans & 743 & -435 & 0 & 0 & 0 & 308 \\
\hline Shares and other equity & 488 & -1 & 0 & 0 & 0 & 488 \\
\hline Insurance technical reserves & 0 & 0 & 0 & 0 & 0 & 0 \\
\hline Financial derivatives & 0 & 0 & 0 & 0 & 0 & 0 \\
\hline Other accounts receivable & 0 & -26 & 0 & 0 & 26 & 0 \\
\hline Monetary gold and SDRs & 0 & 0 & 0 & 0 & 0 & 0 \\
\hline Liabilities & 11,661 & -953 & 0 & 0 & $-1,857$ & 8,852 \\
\hline Special Draw ing Rights & 0 & 0 & 0 & 0 & 0 & 0 \\
\hline Currency and deposits & 26 & 76 & 0 & 0 & 0 & 102 \\
\hline Securities other than shares & 3,865 & -676 & 0 & 0 & 0 & 3,189 \\
\hline Loans & 7,760 & -365 & 0 & 0 & $-1,857$ & 5,538 \\
\hline Shares and other equity & 0 & 0 & 0 & 0 & 0 & 0 \\
\hline Insurance technical reserves & 0 & 0 & 0 & 0 & 0 & 0 \\
\hline Financial derivatives & 0 & 0 & 0 & 0 & 0 & 0 \\
\hline Other accounts payable & 10 & 12 & 0 & 0 & 0 & 22 \\
\hline \begin{tabular}{|l|} 
M emo randum items: \\
Net worth (in percent of GDP) \\
Net financial worth (in percent of GDP) \\
Gross debt \\
Gross debt (in percent of GDP) \\
Net debt \\
\end{tabular} & $\begin{array}{r}-90.1 \\
11,661 \\
108.7 \\
10,156 \\
\end{array}$ & & & & & $\begin{array}{r}-64.2 \\
8,852 \\
78.3 \\
7,739 \\
\end{array}$ \\
\hline
\end{tabular}




\section{ANNEX III. SEYCHELLES: RELATIONS WITH THE FUND}

(As of March 31, 2011)

I. Membership Status: Joined 6/30/1977. Article VIII member since 01/03/1978

II. General Resources Account

Quota

Fund holdings of currency

Reserve Position in Fund

III. SDR Department

Net cumulative allocation

Holdings

\begin{tabular}{|c|c|}
\hline SDR Million & \% Quota \\
\hline 8.80 & 100.0 \\
\hline 29.04 & 329.97 \\
\hline 0.003 & 0.04 \\
\hline
\end{tabular}

$\underline{\text { SDR Million }}$

8.28

7.56

$\%$ Allocations

100.0

91.28

IV. Outstanding Purchases and

Loans:

Stand-by Arrangement

11.00

125.00

Extended Arrangement

9.24

105.00

\section{Financial Arrangements:}

\begin{tabular}{lcccc} 
& & & \multicolumn{2}{c}{ SDR Million } \\
Type & Arrangement & Expiration & Amount Approved & Amount Drawn \\
EFF & Dec 23, 2009 & Dec. 22, 2012 & 19.80 & 9.24 \\
Stand-By & Nov.14, 2008 & Dec. 22, 2009 & 17.60 & 11.00
\end{tabular}

VI. Projected Obligations to the Fund (SDR Million: based on existing use of resources and present holdings of SDRs):

\begin{tabular}{l|ccccc}
\hline & \multicolumn{5}{|c}{ Forthcoming } \\
\hline & $\mathbf{2 0 1 1}$ & $\mathbf{2 0 1 2}$ & $\mathbf{2 0 1 3}$ & $\mathbf{2 0 1 4}$ & $\mathbf{2 0 1 5}$ \\
Principal & & 3.41 & 5.50 & 2.42 & 1.54 \\
Charges/interest & 0.22 & 0.28 & 0.22 & 0.16 & 0.13 \\
Total & 0.22 & 3.69 & 5.72 & 2.58 & 1.67 \\
\hline
\end{tabular}


VII. Implementation of HIPC Initiative: Not applicable

\section{Safeguards Assessments:}

The Central Bank of Seychelles (CBS) was subject to a safeguards assessment with respect to the Stand-By Arrangement for Seychelles. The assessment completed in 2008 found high risks in all areas of the CBS's safeguards framework, and several measures were included in the program. An update assessment in relation to the Extended Fund Facility, which was completed in July 2010, has found that the authorities have implemented most of the measures recommended by the 2008 assessment. In particular, governance oversight at the CBS is now exercised by the Board and management committees, and transparency improved through application of International Financial Reporting Standards and the publication of financial statements that have been audited in accordance with international standards. The draft amendments to the CBS Act, approved by Cabinet in April 2011, will strengthen the legal framework and the independence of the central bank. CBS governance is also being improved through ongoing strengthening of the internal audit and control mechanisms.

\section{Exchange Rate Arrangement:}

The exchange market was liberalized in November 2008 and resulted in elimination of all the restrictions on the making of payments and transfers for current international transactions that are subject to Fund approval under Article VIII Sections 2, 3, and 4. The exchange rate policy is floating. On April 8, 2011 US\$ 1 = SR 12.25 (mid rate).

\section{Article IV Consultations:}

Seychelles is currently under a 24 month consultation cycle. 
XI. Technical Assistance (2008-April 2011):

\begin{tabular}{|c|c|c|c|}
\hline Department & Head of Mission & Subject & Date \\
\hline STA & Mr. Dessart & $\begin{array}{l}\text { Dissemination of GDDS } \\
\text { National Summary Data } \\
\text { Page }\end{array}$ & April 2008 \\
\hline STA & Mr. Armknecht & Consumer price index & April 2008 \\
\hline MCM & Mr. Faulk & $\begin{array}{l}\text { Banking supervision, } \\
\text { drafting of new financial } \\
\text { sector related regulations }\end{array}$ & April 2008 \\
\hline MCM & Mr. Robotham & $\begin{array}{l}\text { National payment systems } \\
\text { project }\end{array}$ & May/June 2008 \\
\hline FAD & Mr. Khemani & $\begin{array}{l}\text { Strengthening expenditure } \\
\text { rationalization and budget } \\
\text { management }\end{array}$ & July 2008 \\
\hline LEG & Mr. Baban & $\begin{array}{l}\text { Exchange Rate and } \\
\text { Exchange Control } \\
\text { Regimes }\end{array}$ & September 2008 \\
\hline FIN & Mr. Hauge & Safeguards assessment & October 2008 \\
\hline FAD & Mr. Krelove & Reform of Tax Policy & $\begin{array}{l}\text { January/February } \\
2009\end{array}$ \\
\hline MCM & Mr. Bartholomew & $\begin{array}{l}\text { Central Bank Capacity } \\
\text { Building after the } 2008 \\
\text { Float }\end{array}$ & February 2009 \\
\hline LEG & Mr. Baban & $\begin{array}{l}\text { Reform of Exchange Rate } \\
\text { Law }\end{array}$ & May 2009 \\
\hline STA & Mr. Jones & $\begin{array}{l}\text { Government Finance } \\
\text { Statistics }\end{array}$ & May 2009 \\
\hline MCM & Mr. Faulk & Bank Supervision & June 2009 \\
\hline $\mathrm{MCM}$ & Mr. Vollan & Monetary Operations & June 2009 \\
\hline MCM & Mr. Robotham & Payments Systems & June 2009 \\
\hline FAD & Mr. Ljungman & $\begin{array}{l}\text { Public Financial } \\
\text { Management }\end{array}$ & July 2009 \\
\hline LEG & Mr. Burns & Fiscal Law & July 2009 \\
\hline FAD & Mr. Kloeden & Tax Administration & September 2009 \\
\hline
\end{tabular}




\begin{tabular}{|c|c|c|c|}
\hline Department & Head of Mission & Subject & Date \\
\hline STA & Mr. Hughes & $\begin{array}{l}\text { National Accounts } \\
\text { Statistics }\end{array}$ & September 2009 \\
\hline $\mathrm{MCM}$ & Mr. Sullivan & Accounting & October 2009 \\
\hline $\mathrm{MCM}$ & Mr. Faulk & Bank Supervision & October 2009 \\
\hline $\mathrm{MCM}$ & Mr. Robotham & Payments Systems & November 2009 \\
\hline STA & Ms. Razin & BoP Statistics & December 2009 \\
\hline MCM & Mr. Bartholomew & $\begin{array}{l}\text { Central Bank Capacity } \\
\text { Building }\end{array}$ & March 2010 \\
\hline $\mathrm{MCM}$ & Mr. Sullivan & $\begin{array}{l}\text { Central Bank Accounting } \\
\text { and Audit }\end{array}$ & October 2010 \\
\hline MCM & Mr. Vollan & Monetary Operations & October 2010 \\
\hline FAD & Mr. Bodin & Tax Policy (VAT) & October 2010 \\
\hline STA & Mr. Hughes & $\begin{array}{l}\text { Quarterly National } \\
\text { Accounts Statistics }\end{array}$ & November 2010 \\
\hline STA & Ms. Razin & BoP Statistics & January 2011 \\
\hline $\mathrm{MCM}$ & Mr. Sullivan & $\begin{array}{l}\text { Central Bank Financial } \\
\text { Reporting and Risk } \\
\text { Management }\end{array}$ & February 2011 \\
\hline FAD & Mr. Kloeden & Tax Reform & February 2011 \\
\hline STA & Mr. Jones & $\begin{array}{l}\text { Government Finance } \\
\text { Statistics }\end{array}$ & March 2011 \\
\hline MCM & Mr. Bartholomew & $\begin{array}{l}\text { Central Bank Capacity } \\
\text { Building }\end{array}$ & April 2011 \\
\hline STA & Ms. Winston & $\begin{array}{l}\text { Producer Price/Industrial } \\
\text { Production Indices }\end{array}$ & April 2011 \\
\hline
\end{tabular}




\section{APPENDIX I}

\section{SEYCHELLES: LETTER OF INTENT}

May 18, 2011

Mr. Dominique Strauss-Kahn

Managing Director

International Monetary Fund

Dear Mr. Strauss-Kahn:

1. The attached Supplementary Memorandum of Economic and Financial Policies (MEFP) describes Seychelles' performance through December 2010, and updates the policies that were laid up in the MEFPs dated $1^{\text {st }}$ December 2009, $1^{\text {st }}$ June 2010 and $3^{\text {rd }}$ December 2010 for the remainder of 2011 and for 2012.

2. We request completion of the third review under our Extended Arrangement (EFF) and the release of the sixth tranche of SDR 3.52 million (40 percent of quota). We have made significant progress in our reforms and the program remains on track. All quantitative performance criteria (PCs) at end-December 2010 were met with margins, including a primary surplus exceeding the target by 1.6 percent of GDP, and monetary developments remain favorable. The structural benchmark scheduled for end-December was observed; we expect that the Cabinet will approve the amendments to the Financial Institutions Act (a structural benchmark for end-April 2011) with a two month delay. Overall, our reforms have delivered a stable, growing economy and a sustainable debt profile.

\section{The EFF continues to accompany a comprehensive medium-term structural} reform strategy aimed at consolidating macroeconomic stability and securing growth; and to guide our macroeconomic policies over the current year. Despite our good progress, developments in food and fuel prices and unanticipated financial support for the national airline require some adjustments in our program targets that do not jeopardize our hardearned macroeconomic stability. Specifically, we request a change in net international reserves, and reserve money targets for end-June and end-December 2011, as well as in the fiscal primary balance target for end-December 2011.

4. We believe that the economic and financial policies set forth in the MEFP are sufficient to ensure that the objectives of the program will continue to be met. We stand ready to take any further measures that may prove necessary to meet our objectives. We will consult with the Fund on the adoption of these measures, and in advance of revisions to the policies contained in the MEFP, in accordance with the Fund's policies on such consultations. We remain committed to full implementation of the recommendations of the Fund's latest safeguards assessment of the Central Bank of Seychelles (CBS).

5. It is expected that the fourth review of the arrangement will be completed by end-September 2011 and that the fifth review be completed by end-March 2012. Financing assurance reviews will continue as long as public debt arrears to external private creditors remain outstanding. 
6. In line with our commitment to transparency, we request that the IMF publish this letter, the MEFP, the technical memorandum of understanding (TMU), and the staff report. We will simultaneously publish these documents in Seychelles.

Sincerely yours,

$$
\text { /s/ }
$$

Danny Faure

Vice President and Minister of Finance

Republic of Seychelles
$/ \mathrm{s} /$

Pierre Laporte

Governor

Central Bank of Seychelles

Attachments: MEFP and TMU 


\section{AtTAChMENT 1 \\ Seychelles: Supplementary Memorandum of Economic ANd Financial Policies FOR 2011}

\section{A. Introduction}

1. This document tracks progress during the first year of our macroeconomic and financial policies and structural reforms under the three-year program supported by an Extended Fund Facility (EFF) arrangement. It updates the MEFP of December 3, 2010, and details our policy commitments through the remainder of 2011, to support completion of the fourth EFF review.

\section{B. Macroeconomic and Financial Performance in 2010}

\section{In 2010, the Seychellois economy started to reap the benefits of its stabilization} efforts, and economic activity recovered strongly. Tourist arrivals increased by 11 percent and foreign direct investment (including a large private residential complex) boosted activity, in particular in construction, beverage manufacturing, electricity, and transportation and telecommunication services. Overall, real GDP growth is estimated to have exceeded 6 percent.

3. The economic recovery took place in a low inflation and stable exchange rate environment. Inflation was almost nil year-on-year. The Seychelles rupee was broadly stable against all currencies, with a slight depreciation against the dollar at the beginning of the year being offset by an appreciation towards the end of the year. Gross international reserves reached 2.2 months of prospective imports at year-end.

4. Our macroeconomic policy objectives for $\mathbf{2 0 1 0}$ have been met. The larger-thananticipated government revenue performance for the year as a whole (4 percentage points of GDP) translated into a fiscal primary balance in excess of the initial budget by 1.6 percent of GDP. Part of the revenue margin was used to release the pressure on our highly compressed expenditure, including a contingency for capital expenditure of about 1 percent, set aside at the beginning of the year. The fiscal surplus (2. 8 percentage points of GDP) and the successful restructuring of our external debt facilitated a rapid decline in our public debt, to 82 percent of GDP at year-end, from 128.6 percent a year earlier.

5. Monetary developments remain broadly favourable. Broad money expansion was contained at 13.7 percent year-on year, slightly higher than initially projected despite reserve money growth in line with program targets. Private sector credit (excluding the impact of a loan reclassification) started to recover from a substantial contraction the year before, while net credit to the government declined, sustained by the rapid domestic debt repayment. This resulted in a significant liquidity overhang which in turn pushed interest rates on treasury bills and CBS intervention rates to low levels: short-term interest rates on 3-month treasury bills went below $1 / 2$ percent while retail lending and deposit rates also edged down. 


\section{Program Implementation}

6. The program remains on track and the program of structural reforms is being implemented broadly as scheduled. All quantitative performance criteria were met at end2010. The VAT bill was submitted to the National Assembly and adopted on December 21, 2010, ahead of schedule for this structural benchmark.

\section{Macroeconomic Outlook in 2011}

7. The outlook for the economy is a continued, albeit slower, recovery in 2011 with increased downside risks. Global growth prospects are not significantly different from those prevailing at the 2011 budget preparation. In particular, European economies (the main markets for our exports) are projected to grow at a modest pace. In view of several large resorts commencing operations, we project nonetheless a strong increase in tourism receipts this year. However, prospects for canned tuna (our second largest export) are less encouraging in part due to the worsening impact of piracy on the supply of tuna to the domestic processing factory and no significant growth is expected in this industry over the medium term. More significantly, our small, highly open economy is confronted with substantial external shocks stemming from international food and fuel prices.

8. These international factors are expected to translate into a slowdown in real GDP growth in 2011 to 4 percent, a rise of consumer price inflation to about $5 \frac{1}{2} 2$ percent by year-end, and a negative impact on the trade balance of about 5 percentage points of GDP compared to last year's projections. Lower FDI-related inflows from the exceptionally high level registered in 2010 will nonetheless reduce the current account to 32.7 percentage points of GDP in 2011, from more than 50 percentage points in 2010.

\section{E. Policies for 2011}

9. We intend to make several policy adjustments to help the economy absorb the global price shocks. Our first line of defence against such shocks will continue to be our flexible exchange rate regime. We are also tightening monetary policy and offsetting the negative impact of the shocks on the budget to mitigate second-round inflation effects, limit balance of payments pressures and stay the course towards debt sustainability.

10. We have designed measures to help households adjust to the price shocks. The rise in domestic prices is expected to be gradual because Seychelles' state-owned suppliers of fuel and basic commodities have adopted cautious purchase and stock strategies that will smooth price increases throughout part of the year. While a full pass-through is being implemented for most fuel prices, measures have been adopted to smooth the price of a few sensitive products. A Stabilization Fund has been set up for this purpose to help the relevant state-owned enterprises absorb the associated costs through reimbursable funds.

\section{F. Fiscal Policy}

11. We remain committed to a sustainable fiscal policy, in line with our strategy to bring public debt below 50 percent of GDP by 2018. Our budgetary performance was consistent with forecasts for the first two months of the year and we plan to offset the fiscal cost of the Stabilization Fund by reducing non-priority capital spending by an equivalent 
amount. Revenue performance is expected to be slightly higher than in the budget, mainly reflecting upward revisions in dividends from public enterprises. However, support to the national airline, which faces serious but temporary financial difficulties and whose bankruptcy would disrupt tourism activity, requires a relaxation of our primary surplus target by 0.5 percentage points of GDP in 2011, to 4.5 percent. This will not significantly affect the prospect of fiscal sustainability.

12. Net disbursements of the Stabilization Fund are capped at SR 50 million (the equivalent of 0.4 percentage points of GDP) in 2011. Its objective is to enable eligible state-owned companies to smooth out price increases for cooking gas prices, as well as electricity and public transportation tariffs. It is expected that any disbursed funds will be reimbursed to government once the pass-through is complete. The use of the Stabilization Fund will be monitored closely on a monthly basis to ensure that disbursements are consistent with the 2011 cap.

13. Progress continues in the implementation of our key capital projects. Two new electricity generators are currently being commissioned by PUC, over 150 housing units have been delivered on Perseverance Island, and the rehabilitation of the Victoria Hospital continues. To make up for envisaged disbursements of the Stabilization Fund, the capital budget for the year will be reduced by SR 50 million by delaying non-priority projects, including. In addition, we are maintaining capital projects amounting to SR 50 million under contingency upon revenue targets for end-June 2011.

14. Air Seychelles is implementing a strategic action plan to eliminate operational losses by year-end, limiting the need for budget support. The financial losses it faced at the end of 2010 were caused by a variety of factors, including the Government's policy of encouraging competition through access to landing rights, higher fuel prices, one-off maintenance charges and currency movements (most costs are in USD whilst revenues are in Euros). In support of the plan, detailed in I52-56, budget funds of up to SR83 million ( $0.7 \%$ of GDP) will be provided in 2011 to help cover expected operational losses and debt servicing costs of government-guaranteed emergency loans contracted with Nouvobanq.

\section{G. Tax and Customs Reform}

\section{Replacing Good and Services Tax (GST) by a single rate Value Added Tax} (VAT) remains a cornerstone of our comprehensive tax policy reform. Over the course last year, we have completed several preparatory steps, including by:

- introducing a new Revenue Administrations Act (Jan 2010);

- eliminating business tax threshold for companies and lowering that of noncompanies (January 2010);

- introducing the Income and Non-Monetary Benefits Tax (July 2010);

- increasing the rate of GST on tourism from 10\% to 12\% (November 2010); a last increase of the GST rate to $15 \%$ on tourism is scheduled for November 2011.

- shifting from a system of administrative assessment of Business Tax to one of self-assessment; and

- harmonizing the income tax rate so that all (national and expatriate) employees pay a flat rate of 15 percent (January 2011). 
Combined, these reforms have broadened the tax base, simplified the system and made it fairer and more equitable. Further modernization of the Seychelles Revenue Commission (SRC) will now be undertaken, with a focus on customs administration.

\section{VAT Introduction}

16. A number of policy decisions were taken in preparation for the implementation of VAT in July 2012, including the approval of VAT legislation in December 2010. Based on stakeholders' feedback ,advice from the IMF February 2011 tax mission and basic underlying principles of tax collection predictability, we have made the following decisions:

i. Petroleum will continue to be subject to the same excise taxes per litre, but will be exempt from VAT to keep the taxation system simple. Petroleum taxes yield over a tenth of total tax revenue, with low compliance and administration costs.

ii. Businesses shall be liable for VAT if their turnover is above a threshold or if they opt for voluntary registration.

iii. The threshold level will be set with the aim of obtaining approximately 1,000 registered taxpayers in the first year. Once the SRC masters the administration of these taxpayers, the threshold will be gradually lowered to cover more businesses.

iv. To limit cash-flow issues, VAT-registered businesses will be allowed to offset the VAT liability on imported capital goods against the input VAT credit to which they are entitled when filing the next return.

v. Rental income which is currently subject to GST will be brought under the Business Tax Act.

Cabinet will approve VAT regulations including rates, exemptions and thresholds by endJune 2011.

17. Administrative preparations are also underway. A resident IMF VAT expert funded by an EU grant - will assist the SRC for a period of 18 months starting in June 2011 and covering the first 6 months of VAT implementation. This expert will assist the SRC in developing skills, procedures, manuals necessary to secure a successful transition to VAT.

\section{Tax and Customs Administration reforms}

18. General administrative reforms in the SRC continue - they are aimed at improving self compliance whilst reducing overall collection costs. To facilitate collaboration between the Tax and Customs departments, the SRC will merge the prevailing three sets of identification numbers into one. The single Tax Identification Number will require the repeal of the Seychelles Business Number Act and amendments in the Revenue Administration Act 2009 and the Seychelles Revenue Commission Act by end-July 2011. Additionally, the SRC is carrying out the following measures to improve overall administration of taxes: 
a. Further to a function-based restructuring of the Domestic Tax Division, the Audit Section now consists of two units which segment the taxpayer population by size and similar characteristics. Thus, the SRC will be able to better understand and mitigate compliance risks, improving revenue collection. Filing requirements differ by size of business to decrease processing workload of the Tax Division and reduce compliance costs; small business will only be required to file and pay taxes on a quarterly basis.

b. In addition to public disclosure through its website and printed brochures, the SRC prepares a weekly section in the national daily newspaper explaining a different tax each week. Staff is sent on short attachments with other tax administrations to better understand and develop tax payer education programmes. The first of these will be to the South African Revenue Service in the first half of 2011.

c. Extra staff is being recruited to facilitate implementation of VAT: 10 by July 2011 and an additional 10 in the first quarter of 2012. It is envisaged that 21 new staff members will join Customs, the first 9 in 2011 and a further 12 in 2012.

d. The SRC is enhancing the role of its IT department to ensure tax administration efficiency and further develop its Client Management System, in close coordination with the Government's IT Department through fortnightly meetings.

19. The Customs Management Act is expected to be approved by the National Assembly by end-June 2011. The bill was submitted to the National Assembly in December 2010 , but the latter requested a number of amendments which are currently being finalized by an ad-hoc commission. The Act will become operational in February 2012, giving sufficient time for the development of supporting regulations, staff training and outreach for importers, agents and the general public.

20. A reform team will be appointed by April 2011 to ensure successful implementation of the Customs reform program. This includes the development of a capacity building plan; the implementation of risk management and post clearance audit procedures; the review and development of standard operating procedures; and overall improvements in ASYCUDA (Customs' IT system) effectiveness.

\section{H. Public Financial Management}

\section{Public Financial Management was further strengthened, as reflected in the} recently completed EU assessment. The Public Expenditure and Financial Accountability (PEFA) assessment concluded by the EU in March 2011 noted remaining weaknesses, notably in the area of medium-term budgeting and legislative scrutiny of the budget law. These are being addressed through the formulation of an action plan. In addition, recommendations of the Auditor-General (AG)'s report for budget year 2009 to improve ministries and departments' procedures are followed up by the Audit Committee (created in April 2010). To strengthen expenditure management, the Public Budget Management Section of the Ministry of Finance has started monitoring line ministries' creditor lists on a monthly basis. The Comptroller General will promptly clear any related payments outstanding to 
continue to ensure the non-accumulation of domestic payment arrears. The Ministry of Finance and PUC are currently engaged in an extensive exercise to verify the utility consumption of line ministries for 2010 and settle any final bills within 30 days of their submission.

22. We will adopt a new Public Finance Act (PFA) to incorporate our reformed budget practices. This law will reflect recent operational improvements in public financial management, most notably in the area of budget formulation, execution, reporting and auditing, in line with Fund recommendations. The law will also extend the coverage of the National Assembly's budgetary oversight to capital expenditure. Given the complexity of this reform we have sought Fund advice on drafting the Bill, which we expect to submit to Cabinet's approval by end September 2011.

23. The revision of the Financial Instructions and the Accounting Manual will be completed by June 2011. The new PFA will also provide the basis for revising the Accounting Standards of Financial Statements, aligning them with the International Public Sector Accounting Standards (IPSAS). By September 2012 we will have the necessary framework in place to ensure accounting practices are in line with international best practice.

\section{We plan to introduce a new chart of accounts that incorporates a functional} classification in the 2012 budget. A special committee, spearheaded by the Financial Planning and Control Division of the Ministry of Finance, and including representatives of the Treasury and the Government's Department of Information, Communications and Technology has submitted a proposal and an action plan to Cabinet.

25. We are introducing medium-term policy-based budgeting, to further improve fiscal planning and accountability. For the 2012 Budget, we will undertake a pilot program comprised of the two largest ministries - health and education. Subject to the results of the pilot, we will extend policy-based budgeting to all agencies' budget preparations from 2013. Implementation of the new system is supported by a training program for government officials responsible for budget implementation, which started in late 2010 with courses on asset management and strategic budgeting.

26. Further progress is being made in treasury management and in the rationalization of public entities. The new budget dependent agencies' re-classification policy has been fully implemented, resulting in a sharp reduction in the number of suspense accounts. As a result, strategic assessments of our public entities are being carried out with the objective of addressing duplication and elaborating a plan for privatization.

27. We will launch a strategic plan to establish a well-targeted and sustainable social security system covering the Social Security Fund (SSF) and the Social Pension Fund (SPF). As part of this plan, we are reforming SSF investment objectives. SSF assets were built from collections under the previous system of Social Security Contributions. By April 2011, we will submit an amendment to the Social Security Act to the National Assembly to formalize our current policy whereby no SSF funds may be used for government expenditure. By end-September 2011 we will submit to Cabinet a strategic plan for the investment of SSF assets, which will outline objectives and modalities of investments and permissible uses for related income. This plan will be formulated by the SSF Board of Trustees, which is also entrusted with oversight of the plan's implementation. Meanwhile, part of SSF deposits at commercial banks will be gradually transferred to the central bank. 


\section{Monetary and Exchange Rate Policies}

28. The Central Bank of Seychelles (CBS) will continue to rely on reserve money targeting as its monetary policy framework. It will maintain Repos as the main instrument to guide short-term interest rates while the Deposit Auction Arrangement (DAA) and the Credit Auction Arrangement (CAA) will be used for fine tuning. The CBS is increasing its range of monetary instruments, such as the recently approved foreign exchange SWAPs, to achieve greater flexibility in market interventions.

29. To absorb excess liquidity and prevent the risk of inflationary pressures from the recent increases in global food and fuel prices, the CBS has tightened monetary policy by raising minimum reserve requirements (MRR) on commercial banks' deposits. MRR on domestic and foreign currency deposits were raised from $10 \%$ to $13 \%$, the former effective April 1, the latter effective June 1 (following three monthly steps). Liquidity tightening, compounded by the gradual transfer of SSF deposits to the CBS, is expected to lead to an increase in T-bill rates that could limit the risks of capital outflows.

30. The CBS and the Ministry of Finance are coordinating their efforts to improve liquidity management. A calendar of weekly Treasury bill issuances is communicated four weeks in advance so as to inform the market on the quantity of paper to be issued. This has helped improve the predictability of the securities market and facilitate banks' liquidity management. Closer collaboration with the SRC and Ministry of Finance on daily government expenditure and revenue collection has also been beneficial in terms of liquidity management.

31. We have decided to reintroduce longer terms government bonds in the domestic market as a first step towards the establishment of a benchmark yield curve. The absence of a yield curve is a barrier to financial sector development. We are requesting TA from the IMF to assist us in developing domestic securities markets, including the reintroduction of the secondary market and a primary dealership system.

32. We remain committed to the floating exchange rate regime. This regime continues to function well and the Seychelles rupee has remained resilient in the face of global currency volatility. The CBS will only intervene in the foreign exchange market to smooth out excessive volatility, to ensure orderly market conditions, and, if needed, to build up international reserves in line with program objectives. We stand ready to consider additional recommendations from the IMF to further improve the functioning of the foreign exchange market, in particular the inter-bank market. We will introduce a code of conduct for foreign exchange and money market participants in late 2011.

33. The CBS continues to target a gross external reserve target of 3-month import coverage. It considers this threshold as a cushion against Seychelles' relative openness to the world economy and vulnerability to external shocks. At end-2010, we reached 2.2 months of import cover and remain on track to meet this target in a reasonably short period. To help absorb global price shocks, however, the reserve accumulation objectives for 2011 have been slightly adjusted down.

\section{Given its internal capacity constraints, the CBS will maintain a prudent} approach to its reserve management program, limiting the use of external parties to manage its reserve asset to the World Bank (RAMP program) and the Bank for International Settlement. The CBS Investment and Risk Implementation Committee will finalize proposals 
and enter into formal arrangements with preferred institutions during the second quarter of 2011. Besides their more favorable terms for investing a portion of external reserves, these institutions will also provide technical assistance on reserve management to the CBS.

\section{J. CBS Operations and Governance}

35. The first phase in the implementation of an integrated (core) banking system went live on December 01, 2010 as planned. The CBS can now provide all banking services on a single IT platform, which has greatly enhanced the efficiency of its operations and significantly reduced operational risks stemming from different types of software. We have now started a second phase to integrate other CBS operations, including payroll, HR management and inventory management.

36. The modernization of the national payment system is gathering momentum. Several major steps were taken in 2010, including the formalization of new clearing house rules and the adoption of the National Clearing and Settlement system Act. The successful implementation of the new core banking system, which provides straight-through-processing of interbank and Government transaction on a real time basis, will bring additional benefits to the payment system in 2011. This new system provides the platform for implementing an Electronic Clearing House and introducing a local Switching System to facilitate the settlement of rupee ATM/POS transactions. The CBS will continue to promote electronic funds transfer and cards. A real time gross settlement system will be introduced by end- 2012 .

37. The capital of the CBS will be increased in 2011, in line with the CBS Act. The recapitalization of the CBS through t-bill issuance on December 15, 2010 brought the capital of the central bank to 10 percent of end-2009 monetary liabilities, enhancing its portfolio of tradable securities for open market operations. A transfer of SR 13.4 million from 2010 distributable profits will bring capitalization of the CBS to 9.21 percent of end-2010 monetary liabilities.

38. The CBS legal framework has been further strengthened, addressing vulnerabilities and enhancing independence. In line with the 2010 IMF Safeguards Assessment a set of amendments to the CBS Act 2004 will be submitted for Parliament's approval by end-June 2011. Among the key amendments are provisions which clarify the terms of lending to Government: any such lending above the stipulated limit will be subject to punitive terms (form of higher above-market interest rates and shorter repayment periods) except in cases of force majeure. Other provisions include (i) a strict prohibition on receiving or seeking outside instructions for CBS employees and board members; (ii) an explicit reference to Governor and Deputy Governor terms of office of six years; and (iii) due process in the event of dismissal, including for the Governor, Deputy Governor and Board members.

39. CBS governance is being improved through further strengthening of internal control and audit mechanisms. In February 2011, the CBS Board approved a risk policy and a framework for risk management and established a Risk Implementation Committee. This committee has since merged with the Investment Committee to enhance efficiency and stimulate synergies. In the coming months the CBS will put in place a new structure that will provide clear segregation of duties relating to reserve management activities of front, middle and back office functions. Internal audit has been established as a fully fledged CBS division. 


\section{K. Financial Sector Reforms}

40. We are initiating several measures to enhance competition and promote the deepening of the underdeveloped financial sector. According to the 2010 Article IV consultation report, Seychelles banks appear to have a higher return on equity than their peers, which could imply a lack of competition. A recent study by the CBS identified several impediments to the development of commercial bank credit to the private sector including (i) high switching costs by banks; (ii) inadequate disclosure by financial institutions; (iii) absence of a credit rating system; (iv) excessive costs and lengthy procedures for registration of loan and other bank-related documents; and (v) lengthy procedures for resolution of financial disputes. To address these shortcomings, the Ministry of Finance has reduced the charges and timeframe for document registration; it will work with the Judiciary to put in place a faster system for resolution of financial disputes; and it will propose some amendments to the Financial Institutions Act (FIA) (see II41 below). In addition, the CBS will introduce measures to minimize switching costs, and will implement a financial literacy campaign to enhance public awareness and encourage informed decisions to reinforce competitive pressures on banks.

41. The proposed amendments to the FIA, to be approved by Cabinet by June 2011, will also strengthen the regulatory framework and enhance competition. They will allow for (i) the introduction of new financial products (e.g., leasing, hire purchase) and Islamic banking (to be licensed and regulated by the CBS); and (ii) the establishment of a Credit Reference Bureau to allow information sharing on potential lenders between banks. The proposed amendments will also give the CBS extra regulatory powers, including to:

(i) enhance disclosure of banks' terms and conditions (to ensure greater transparency toward the public); (ii) impose fines; and (iii) regulate certain categories of fees/charges, especially where these are deemed to inhibit competition in the financial sector.

42. Our endeavor to further develop the financial sector will be boosted by the establishment of a stock exchange in Seychelles. Whilst the Seychelles market may not solely justify the existence of a stock exchange we are confident that the latter could attract sufficient offshore business, including from the Indian Ocean, South Asia, the Middle East and Africa. Government has decided to tender out the process for establishing the exchange and proposals will be invited in the coming months. A national committee will be established with the task of selecting the most suitable candidate based on technical and financial criteria to ensure utmost transparency in process.

\section{The CBS continues to work towards developing a risk-based supervision}

framework which was initiated with IMF technical assistance. Negotiations are underway with IT companies to develop a Bank Supervision Application forming the basis for conducting offsite supervision under the CAELS approach. On-site supervision of institutions under the purview of CBS will continue as per the scheduled timeframe, in line with the CAMELS banks rating system. In the context of this risk-based approach the CBS will require banks to submit business plans on an annual basis by end-2011, enhancing macroprudential supervision of financial institutions.

44. Supervision of the Housing Finance Company (HFC) and the Development Bank of Seychelles (DBS) is progressing well. The CBS has started the on-site examination of DBS and a final report is expected to be ready by end of May 2011. Offsite supervision of DBS is currently underway. The on-site examination of HFC is expected to start in August 2011, meanwhile training is being provided to HFC to support the submission of prudential reports to the CBS on a regular basis. 
45. The CBS has advanced considerably in developing the appropriate safeguards for financial sector stability. A first draft of the crisis preparedness binder has been completed and circulated to key stakeholders for comments, addressing identified gaps. The draft has been sent to Toronto Centre for its review in April 2011.

46. Seychelles will continue its efforts to promote transparency and stability of the offshore financial sector. Work is under way to address several legal and regulatory weaknesses in the area of exchange of information that had been identified by a recent peer review of the Global Forum on Transparency and Exchange of Information for Tax Purposes (II48). In addition, the CBS will seek to start negotiations for its membership within the Offshore Group of Banking Supervisors.

\section{Substantial legislative changes are being prepared to improve the tax transparency of our offshore sector:}

- A new Companies Act will unify the existing "dual" system of the Companies Act 1972 and International Business Companies Act 1994, and provide a stronger regulatory framework, including for international business companies.

- A Revenue Administration Regulation under the Revenue Administration Act will cater for Seychelles obligations to exchange information with third countries.

- Additional Tax Information Exchange Agreements are being finalized, including with the Netherlands and six Nordic countries.

- A new International Corporate Service Providers law will clarify the authorities' ability to request documents and information (ensuring that all relevant information required is available on offshore entities).

- A Financial Services Commission will be legally established to reflect the increased responsibility of SIBA in enforcing transparency of the offshore sector.

48. We have made important strides in implementing recommendations of the 2006 Mutual Evaluation of Seychelles the Anti-Money Laundering (AML) and Counter Financing of Terrorism (CFT) regime by the Eastern and Southern Africa Anti-Money Laundering Group (ESAAMLG). In particular, in 2008, the Financial Intelligence and Assets Recovery Unit (FIU) was established and attributed investigative powers and duties. Staffing in FIU has been recently increased, enabling the FIU to ensure that reporting entities comply with the requirements of the AML Act and the Prevention of Terrorism Act 2004 by means of on-site inspections. The FIU has also applied to become a member of the Egmont Group and has assisted law enforcement agencies from seventeen countries to date.

49. The CBS is committed to encouraging a sound and efficient insurance sector that promotes policyholder confidence. To this end, various capacity building initiatives are underway. A workshop on insurance supervision, facilitated by Commonwealth, was held in March 2011. In addition, the FIRST Initiative has agreed to provide TA to the CBS to strengthen insurance supervision, including by reviewing the regulatory framework and preparing manuals for conducting examination of insurance and insurance-related companies. 
Table 1: Schedule of Actions for 2011

\begin{tabular}{|l|l|}
\hline Submit first Draft of Crisis Binder to Toronto Centre & Apr-11 \\
\hline Submit FIA amendments to Cabinet of Ministers & Jun-11 \\
\hline $\begin{array}{l}\text { Start negotiations for membership within the Offshore Group of } \\
\text { Banking Supervisors }\end{array}$ & Jul-11 \\
\hline Implementation of Bank Supervision Application & Dec-11 \\
\hline Require banks to submit business plans & Dec-11 \\
\hline $\begin{array}{l}\text { Review regulatory framework of insurance sector, preparation } \\
\text { of manuals for insurance supervision }\end{array}$ & Dec-11 \\
\hline \begin{tabular}{l} 
Conduct onsite examinations of DBS and HFC \\
\hline
\end{tabular} & Dec-11 \\
\hline
\end{tabular}

\section{Public Enterprises}

50. In view of recently increased quasi-fiscal risks, public enterprise monitoring will be reinforced. In April 2011, a revised policy to formalize the selection criteria of Board members was submitted to Cabinet; a second policy revision is expected to be approved by Cabinet in July to align Board remuneration with the level of responsibility and work entailed. A privatization plan for non-strategic enterprises which do not serve policy goals is on track for September 2011.

\section{Air Seychelles}

51. Air Seychelles' operations have been crucial to the tourism industry, which is the mainstay of the economy. Although its share of the market has been declining, Air Seychelles still transports more than half of all tourist visitors to the country. In contrast to many countries in the OECD and the Indian Ocean, Seychelles has allowed Gulf-based carriers to increase flight frequencies in 2010 and 2011, an action driven by Government's desire to encourage the growth of tourist arrivals and its commitment to openness and competition. The Government is thus committed to ensure any shocks affecting the airline do not disrupt the continuous flow of visitors to the country.

\section{The financial position of the national airline deteriorated in $\mathbf{2 0 1 0}$ due to a} number of pricing and contractual issues. It was also affected by greater competition, fuel price increases, one-off maintenance charges and adverse currency movements. In December and January the Government provided a guarantee on loans of SR 225 million (2\% of GDP) to re-finance the airline's debt, which are to be repaid over a five-year period.

53. Bold steps have been taken to eliminate the company's losses and limit the drain on the Government's current budget. The Government replaced the company's management and Board in February 2011 and charged it with eliminating losses whilst focusing on the core business (i.e. providing direct air access for visitors to the Seychelles). The new management team has prepared a strategic action plan and is taking immediate remedial action. 
54. The central elements of the plan are eliminating loss-making routes, streamlining and modernizing the fleet, improving the terms of Air Seychelles' code-share arrangement with Air France on the critical Seychelles-Paris route, and passing on fuelprice increases. Although the airline enjoys a high load factor (number of seats sold per flight) on most of its routes, its cost base is high due in part to an ageing fleet with high maintenance and lease costs. Low overall utilization of the long-haul fleet is also a problem.

55. The government is confident that a restructured Air Seychelles can be placed on a sound financial footing so as to minimize the cost of its recapitalization, within one year. Internal reforms undertaken in the next few months will be crucial and the Ministry of Finance is closely monitoring the situation and will report to the IMF on a quarterly basis.

$P U C$

56. Our commitment to improving our capacity to invest in public infrastructure has not abated, particularly regarding urgent needs in the utilities sector. BADEA will finance expert, who would carry out the study on PUC tariffs and mapping out the latter in consistency with full cost recovery and meeting future investment needs. This is on course for completion by end-July 2011. Additionally we will reinstate tariff adjustments to fuel price variations by end-September 2011, with a view to eliminating PUC's operational losses and any further recourse to the Stabilization Fund.

\section{Public Financial Institutions}

57. We have taken major steps toward our review of the privatization of the two State-owned banks, Nouvobanq and Seychelles Savings Bank (SSB). The Seychelles Government has met the International Finance Corporation's advisory services with a view to bring the review process of the Government's shares in Nouvobanq to completion before end-2011. The IFC is also considering subscribing to a capital augmentation of 20 percent as a way to facilitate its privatization.

58. Government has offered 40 percent of SSB's shares to the private sector beginning April 2011. The shares will be tradable. This initial sale of shares was offered to the general public, with a priority given to the bank's account holders and employees.

59. Measures are being implemented to enhance the DBS's lending capacity and governance procedures. In recent months, DBS offered more affordable rates (8.5 percent) and more flexible terms, which increased demand for its loans. This in turn has put excessive strain on the financial position of the bank, which currently has insufficient funds to satisfy its portfolio of approved loans despite the issuance of government-guaranteed bonds on the domestic financial market at 6\% (a rate which provides enough spread to cover the bank's operating costs despite the lower lending rates). In March 2011, the DBS Board stopped the refinancing of existing loans, limited the size of loans to SR6 million (with additional lending to be done on a pari passu basis with commercial banks) to curb demand. Several other actions will be implemented in the second half of 2011 such as establishing minimum liquidity buffers and enhancing governance procedures.

60. A new law governing DBS to reflect its new mandate will be introduced in early 2012. The emphasis will be on small and medium-sized enterprises (SMEs) in targeted, development-oriented sectors, with high risk projects that commercial banks would generally not be willing to finance. Given the high risk nature of such projects interest rates could at times exceed those offered by commercial banks. 
61. As part of the action plan we plan to adopt by end-June 2010 for house financing policy, we are also working on a strategy to modernize HFC. The action plan will in particular limit the role of the public sector within the constraints of the significant pent-up demand for housing and limited commercial bank financing for lower income applicants. The strategy will be informed by the recommendations of the FIRST initiative's strategic review. It was drawn up and presented to the management and the HFC Board in April 2011prior to Government approval.

\section{Total and External Public Debt}

62. Our revised public debt management strategy policy, which was adopted together with the current budget, entails a gradual reduction of our total public indebtedness to 50 percentage points of GDP by 2018. Despite the recent issuance of government guarantees in relation to the domestic loans extended to Air Seychelles and the bonds issued locally by DBS, and a less favorable macroeconomic context, total public debt is projected to decline to 76.2 percent at end-2011, from 82.3 percent at end-2010. Unless force majeure, we do not plan to issue additional government guarantees on domestic loans in 2011.

63. Our external debt restructuring is close to completion. Seychelles remains in discussions with two official bilateral non-Paris creditors (Abu Dhabi and India) on the restructuring of outstanding claims and expects to conclude shortly bilateral agreements with two remaining commercial creditors. In addition, we are seeking the finalization of the two remaining bilateral agreements with Paris Club creditors. We are committed to meet the program limits on contracting new government or government-guaranteed external loans as set out during the last program review.

\section{N. Statistics}

64. We have adopted a strategic plan to achieve compliance with SDDS and BPM6, based on the recommendations of the IMF and other external advisors. In September 2011 we will commence publication of quarterly and annual GDP statistics under the SDDS framework, as well as improve labor market statistics. By December 2012 we will compile the production index on a quarterly basis using the quarterly GDP indicators. In 2012 we will redevelop data sources and estimation methods in response to the introduction of the VAT. The new chart of accounts will facilitate the reporting of fiscal data in compliance with the Government Finance Statistics Manual 2001 (GFSM 2001).

65. Other initiatives include our participation, for the first time, in the International Comparison Program being coordinated by the African Development Bank and efforts to improve our data sources by drawing on a wider range of taxation and survey data. We are also enhancing our institutional capacity through increased staffing, training, and information dissemination, including through participation in IMF training courses.

\section{O. Program Monitoring}

66. The program will continue to be monitored by semi-annual reviews, with semi-annual quantitative performance criteria and disbursements, in line with the MEFP of May 16, 2010. The quantitative performance criteria for 2011 are shown in Table 2. The structural benchmarks are shown in Table 3. The attached revised Technical Memorandum of Understanding (TMU) defines the quantitative performance criteria, indicative targets, and adjusters under the program. 
67. Seychelles will avoid introducing new exchange restrictions, multiple currency practices, or bilateral payment agreements in contradiction with Article VIII of the IMF's Articles of Agreement and imposing any import restrictions for balance of payments reasons.

68. We stand ready to adopt any additional measures, in consultation with IMF staff, which may become necessary to ensure program success. 
Table 2. Seychelles: Quantitative Performance Criteria Under the Extended Arrangement, December 2010-December 2011 (Millions of Seychelles rupees; end-of-period)

\begin{tabular}{|c|c|c|c|c|c|c|c|c|c|c|c|c|}
\hline & \multicolumn{3}{|c|}{2010} & \multicolumn{9}{|c|}{2011} \\
\hline & \multicolumn{3}{|c|}{ December } & \multicolumn{3}{|c|}{ March } & \multicolumn{2}{|l|}{ June } & \multicolumn{2}{|c|}{ September } & \multicolumn{2}{|c|}{ December } \\
\hline & Program & Adjusted & Actual & $\begin{array}{c}\text { Indicative } \\
\text { target }\end{array}$ & Adjusted & Prel. & $\begin{array}{l}\text { Performance } \\
\text { Criteria }\end{array}$ & Revised & $\begin{array}{c}\text { Indicative } \\
\text { target }\end{array}$ & Revised & $\begin{array}{l}\text { Performance } \\
\text { Criteria }\end{array}$ & Revised \\
\hline \multicolumn{13}{|l|}{ Performance criteria } \\
\hline Net international reserves of the CBS, millions of U.S. dollars (floor) ${ }^{1}$ & 168 & 148 & 204 & 189 & 191 & 210 & 192 & 204 & 202 & 203 & 213 & 199 \\
\hline Reserve money (ceiling) & 1,753 & $\ldots$ & 1,746 & 1,804 & $\ldots$ & 1,772 & 1,824 & 1,755 & 1,844 & 1,757 & 1,862 & 1,765 \\
\hline Primary balance of the consolidated government (cumulative floor) ${ }^{2}$ & 823 & & 1,031 & 142 & $\ldots$ & 419 & 240 & 240 & 515 & 462 & 602 & 536 \\
\hline $\begin{array}{l}\text { The contracting or guaranteeing of new external debt by the public sector } \\
\text { (Millions of U.S. dollars; cumulative ceiling) }{ }^{2}\end{array}$ & 37 & $\cdots$ & 7 & 4 & $\ldots$ & 0 & 33 & 33 & 46 & 46 & 47 & 47 \\
\hline $\begin{array}{l}\text { The contracting or guaranteeing of new short-term external debt by the public sector } \\
\text { (Millions of U.S. dollars; cumulative ceiling) }{ }^{2}\end{array}$ & 0.0 & $\ldots$ & 0.0 & 0.0 & $\ldots$ & 0.0 & 0.0 & 0.0 & 0.0 & 0.0 & 0.0 & 0.0 \\
\hline The accumulation of external payments arrears by the public sector (ceiling) ${ }^{3}$ & 0.0 & $\ldots$ & 0.0 & 0.0 & $\ldots$ & 0.0 & 0.0 & 0.0 & 0.0 & 0.0 & 0.0 & 0.0 \\
\hline The accumulation of domestic payment arrears by the government (ceiling) & 0.0 & & 0.0 & 0.0 & $\ldots$ & 0 & 0.0 & 0.0 & 0.0 & 0.0 & 0.0 & 0.0 \\
\hline \multicolumn{13}{|l|}{ Memorandum items: } \\
\hline Net external non project financing (millions of U.S. dollars; cumulative) ${ }^{2}$ & 31.8 & $\ldots$ & 11.4 & -1.4 & $\ldots$ & 0.7 & 0.0 & -0.6 & 2.1 & 6.8 & 5.5 & 1.6 \\
\hline \multicolumn{13}{|l|}{ Program accounting exchange rates } \\
\hline SR/US\$ (end-of-quarter) & 11.75 & & $\ldots$ & 11.75 & $\ldots$ & $\ldots$ & 12.30 & 12.15 & 12.30 & 12.15 & 12.30 & 12.15 \\
\hline US $\$ /$ Euro (end-of-quarter) & 1.27 & $\ldots$ & $\ldots$ & 1.27 & $\ldots$ & $\ldots$ & 1.36 & 1.34 & 1.36 & 1.34 & 1.36 & 1.34 \\
\hline US $\$ /$ UK pound (end-of-quarter) & 1.48 & $\ldots$ & $\ldots$ & 1.48 & $\ldots$ & $\ldots$ & 1.57 & 1.56 & 1.57 & 1.56 & 1.57 & 1.56 \\
\hline US\$/SDR (end-of-quarter) & 1.49 & $\ldots$ & $\ldots$ & 1.49 & $\ldots$ & $\ldots$ & 1.56 & 1.49 & 1.56 & 1.49 & 1.56 & 1.49 \\
\hline
\end{tabular}

Sources: Seychelles authorities and IMF staff estimates and projections.

The floor will be adjusted downward (upward) for any shortfall (excess) in external nonproject financial support from that assumed in the program.

2 Cumulative net flows from the beginning of the calendar year; includes external non-project loans and grants net of external debt service payments.

${ }^{3}$ The nonaccumulation of new external payment arrears constitutes a continuous performance criterion. Excludes arrears for which a rescheduling agreement is sought. 
Table 3: Structural Benchmarks, 2010-11

\begin{tabular}{|c|c|c|c|}
\hline Measure & Target Date & $\begin{array}{l}\text { Macroeconomic } \\
\text { Rationale }\end{array}$ & Note \\
\hline $\begin{array}{l}\text { Cabinet approval of the } \\
\text { amendments to Financial } \\
\text { Institutions Act as described in } \\
\text { MEFP, II } 41\end{array}$ & End-April 2011 & $\begin{array}{l}\text { To strengthen competition in } \\
\text { the banking sector and } \\
\text { improve risk management. }\end{array}$ & $\begin{array}{l}\text { Not met. Moved to } \\
\text { end-June2011. }\end{array}$ \\
\hline $\begin{array}{l}\text { Adopt an action plan for house } \\
\text { financing policy that limits the } \\
\text { role of the public sector (MEFP, } \\
\text { 【61) }\end{array}$ & End-June 2011 & $\begin{array}{l}\text { To reduce fiscal risks, } \\
\text { strengthen competition, and } \\
\text { promote development of the } \\
\text { banking system. }\end{array}$ & \\
\hline $\begin{array}{l}\text { Launch a strategic plan for the } \\
\text { reform of the social security } \\
\text { system (MEFP, I27) }\end{array}$ & End-June 2011 & $\begin{array}{l}\text { To establish well-targeted and } \\
\text { sustainable social security } \\
\text { system. }\end{array}$ & \\
\hline $\begin{array}{l}\text { Cabinet approval of VAT } \\
\text { regulations, including rates, } \\
\text { exemptions, and thresholds } \\
\text { (MEFP, đ16) }\end{array}$ & End-June 2011 & $\begin{array}{l}\text { To modernize the tax system } \\
\text { and remove tax distortions. }\end{array}$ & \\
\hline $\begin{array}{l}\text { Commission and complete a study } \\
\text { on optimal tariffs for utilities } \\
\text { (MEFP, I56) }\end{array}$ & End-July 2011 & $\begin{array}{l}\text { To ensure cost recovery and } \\
\text { long-term sustainability of } \\
\text { utilities. }\end{array}$ & \\
\hline $\begin{array}{l}\text { Develop a privatization plan for } \\
\text { nonstrategic public enterprises, } \\
\text { which do not serve public policy } \\
\text { goals (MEFP, I[50) }\end{array}$ & $\begin{array}{l}\text { End-September } \\
2011\end{array}$ & $\begin{array}{l}\text { To reduce the role of the state } \\
\text { in the economy, improve } \\
\text { corporate governance and } \\
\text { minimize fiscal risks. }\end{array}$ & \\
\hline $\begin{array}{l}\text { Cabinet approval of a new Public } \\
\text { Finance Bill extending the } \\
\text { National Assembly's oversight on } \\
\text { capital expenditure budget } \\
\text { (MEFP, I22) }\end{array}$ & $\begin{array}{l}\text { End-September } \\
2011\end{array}$ & $\begin{array}{l}\text { To strengthen public finance } \\
\text { management. }\end{array}$ & \\
\hline $\begin{array}{l}\text { Reinstate the electricity tariff } \\
\text { adjustment for fuel price variation } \\
\text { (MEFP, } \llbracket 556 \text { ) }\end{array}$ & $\begin{array}{l}\text { End-September } \\
2011\end{array}$ & $\begin{array}{l}\text { To reduce losses of the public } \\
\text { utility company. }\end{array}$ & \\
\hline
\end{tabular}




\section{ATtaChment 2}

\section{SEYCHELLES: TECHNICAL MEMORANDUM OF UNDERSTANDING}

1. This technical memorandum of understanding presents the definitions of variables included in the quantitative performance criteria and indicative targets set out in the memorandum of economic and financial policies (MEFP), the key assumptions, and the reporting requirements of the Government and the Central Bank of Seychelles (CBS) needed to adequately monitor economic and financial developments. The quantitative performance criteria and indicative targets, and the benchmarks for 2011 are listed in Tables 2 and 3 of the MEFP, respectively.

\section{Quantitative Performance Criteria}

\section{A. Net International Reserves of the CBS (Floor)}

\section{Definition}

2. Net international reserves (NIR) of the CBS are defined for program monitoring purposes as reserve assets of the CBS, minus reserve liabilities of the CBS (including liabilities to the IMF). Reserve assets of the CBS are claims on nonresidents that are readily available (i.e., liquid and marketable assets, free of any pledges or encumberments and excluding project balances and blocked or escrow accounts, and bank reserves in foreign currency maintained for the purpose of meeting the reserve requirements), controlled by the CBS, and held for the purpose of intervening in foreign exchange markets. They include holdings of SDRs, holdings of foreign exchange, demand and short-term deposits at foreign banks abroad, fixed-term deposits abroad that can be liquidated without penalty, and any holdings of investment-grade securities. Reserve liabilities of the CBS comprise liabilities to nonresidents contracted by the CBS, any net off-balance-sheet position of the CBS (futures, forwards, swaps, or options) with either residents or nonresidents, including those to the IMF.

\section{Calculation method}

3. For program monitoring purposes, reserves assets and liabilities at each test date, must be converted into U.S. dollars using the end of period exchange rates assumed in the program.

\section{MONITORING AND REPORTING}

4. At each program test date, the quarterly net international reserves data submitted by the CBS to the IMF will be audited by the CBS external auditors in accordance with International Standards on Auditing, to ensure conformity with the program definition and calculation methods. Reports will be submitted to the CBS, with a copy to the IMF, no later than two months after each test date. 


\section{Adjusters}

5. The floor on the CBS's NIR will be adjusted upward (downward) by the amount by which the external non-project loans and cash grants exceeds (falls short of) the amounts assumed in the program (MEFP Table 2). The floors will also be adjusted upwards (downwards) by the amount that external debt service payments fall short (exceed) the amounts assumed in the program.

\section{B. Reserve Money (Ceiling)}

\section{Definition}

6. Reserve money is equivalent to currency issued and deposits held by financial institutions at the central bank (bank reserves), including those denominated in foreign currencies. Evaluation of performance of reserve money with respect to the program ceiling will be done at the program accounting exchange rate.

\section{Monitoring and reporting}

7. For each program test date, the quarterly reserve money data submitted by the CBS to the IMF will be audited by the CBS' external auditors in accordance with International Standards on Auditing, to ensure conformity with the program definition. Reports will be submitted to the CBS, with a copy to the IMF, no later than two months after each test date.

\section{Primary Balance of the Consolidated Government (Cumulative Floor)}

8. The consolidated government primary balance from above the line on a commitment basis is defined as total consolidated government and social security fund revenues (excluding privatization and long-term lease income receipts) less all noninterest (primary) expenditures of the government and social security fund.

\section{Public External Debt (Ceiling)}

9. The ceiling applies to the contracting or guaranteeing of new external liabilities by the public sector (including the central government, the CBS, and all public agencies and parastatals for operations that are not directly linked to commercial activities). The ceiling does not apply to the use of Fund resources, operations related to external debt restructuring; normal import related credits; purchases of treasury securities by nonresidents; or borrowing by parastatals in the conduct of normal commercial operations. The non-zero ceilings on the contracting or guaranteeing of external debt are to allow for normal public project finance and program support from multilateral institutions exclusively. Debt shall be valued in U.S. dollars at program exchange rates. A zero sub-ceiling on short-term external debt applies continuously to the contracting or guaranteeing of short-term external debt by the public sector, with an original maturity of up to and including one year.

10. For the purposes of this performance criterion, the definition of debt is set out in Executive Board Decision No.6230-(79/140), Point 9, as revised on August 31, 2009 (Decision No. 14416-(09/91)).. Debt is understood to mean a current, non contingent liability, 
created under a contractual arrangement through the provision of value in the form of assets (including currency) or services, and which requires the obligor to make one or more payments in the form of assets (including currency) or services, at some future points in time; these payments will discharge the principal and/or interest liabilities incurred under the contract. The ceiling on contracting official and officially guaranteed external debt includes all form of debt, including:

a. loans, i.e., advances of money to the obligor by the lender made on the basis of an undertaking that the obligor will repay the funds in the future (including deposits, bonds, debentures, commercial loans, and buyers credits) and temporary exchanges of assets that are equivalent to fully collateralized loans under which the obligor is required to repay the funds, and usually pay interest, by repurchasing the collateral from the buyer in the future (such as repurchase agreements and official swap arrangements);

b. $\quad$ suppliers credits, i.e., contracts where the supplier permits the obligor to defer payments until some time after the date on which the goods are delivered or services are provided; and,

c. leases, i.e., arrangements under which property is provided which the lessee has the right to use for one or more specified period(s) of time that are usually shorter than the total expected service life of the property, while the leasor retains title to the property. The debt is the present value (at the inception of the lease) of all lease payments expected to be made during the period of the agreement excluding those payments that cover the operation, repair, or maintenance of the property.

d. Arrears, penalties, and judicially awarded damages arising from the failure to make payment under a contractual obligation that constitutes debt are debt.

\section{E. External Arrears of the Public Sector}

11. The nonaccumulation of arrears to external creditors will be a continuous performance criterion under the program. External payments arrears for program monitoring purposes are defined as the amount of external debt service due and not paid within the contractually agreed period, including contractual and late interest. Arrears resulting from the nonpayment of debt service for which a clearance framework has been agreed or a rescheduling agreement is sought are excluded from this definition.

\section{F. Domestic Arrears of Government}

12. The nonaccumulation of budget expenditure arrears will be a performance criterion under the program and will be measured on net basis from the beginning of a calendar year. Budget expenditure arrears are defined as the sum of (1) any invoice that has been received by a spending agency from a supplier of goods, services, and capital goods delivered and verified, and for which payment has not been made within the contractually agreed period, or in the absence of a grace period, within 30 days; (2) unpaid wages, pensions, or transfers, pending for longer than 30 days to domestic or foreign residents, irrespective of the currency 
denomination of the debt; (3) debt service payment on domestic debt of the government or guaranteed by the government that has not been made within the contractually agreed period.

\section{Data and Information}

13. The Seychelles authorities (government and CBS) will provide Fund staff with the following data and information according to the schedule provided.

\section{The CBS will report}

Weekly (within one week from the end of the period)

- $\quad$ Reserve money.

- Foreign exchange reserves position.

- A summary table on the foreign exchange market transactions.

- The results of the liquidity deposit auctions, primary Treasury bill auctions, and secondary auctions.

Monthly (within 4 weeks from the end of the month)

- The monetary survey in the standardized report form format.

- The foreign exchange cash flow, actual and updated.

- Financial soundness indicators.

- Stock of government securities in circulation by holder (banks and nonbanks) and by original maturity and the debt service profile report.

\section{The Ministry of Finance will report}

Monthly (within 2 weeks from the end of the month):

- Consolidated government operations on a commitment basis and cash basis in the IMF-supported program format.

- The detailed revenues and expenditures of the central government and social security fund.

- Accounts of the public nonbank financial institutions.

- Import and export data from the customs department.

- Public debt report.

- Statements of Stabilization Fund operations

- $\quad$ Consolidated creditors schedule on domestic expenditure arrears of the government.

Quarterly (within a month from the end of the quarter):

- Financial statements of Air Seychelles

The government and CBS will consult with Fund staff on all economic and financial measures that would have an impact on program implementation, and will provide any additional relevant information as requested by Fund staff. 
Press Release No. 11/213

FOR IMMEDIATE RELEASE

June 3, 2011
International Monetary Fund

Washington, D.C. 20431 USA

\section{IMF Completes the Third Review Under the Extended Arrangement for Seychelles}

The Executive Board of the International Monetary Fund (IMF) has completed the third review under the three-year Extended Arrangement (EFF), which became effective on December 23, 2009 in the amount of SDR 19.8 million (see Press Release No. 09/472). The completion of the review makes available a disbursement of SDR 3.52 million (about US\$5.6 million). The Board's decision was taken on a lapse of time basis. ${ }^{1}$

In 2010, the Seychelles economy recovered strongly from the debt and balance of payments crisis in 2008, followed by last year's global recession. Economic growth was driven by exceptionally high foreign direct investment and a rebound in tourism. Inflation remained near zero, and the country continued to rebuild its international reserves and reduce public debt. The economic program supported by the Extended Fund Facility remains on track and all quantitative performance criteria at end-December 2010 were met.

In 2011, the policy mix under the program has been adjusted in response to the global commodities price shock and the recent financial difficulties of Air Seychelles. Fiscal policy will be slightly looser to accommodate additional priority spending, while maintaining the targeted progress toward external stability and debt sustainability. Structural reforms will focus on strengthening the financial sector through the modernization of its legal and regulatory framework and the restructuring and/or privatization of several state-owned financial institutions.

\footnotetext{
${ }^{1}$ The Executive Board takes decisions under its lapse of time procedure when it is agreed by the Board that a proposal can be considered without convening formal discussions.
} 